TRANSACTIONS OF THE

AMERICAN MATHEMATICAL SOCIETY

Volume 363, Number 8, August 2011, Pages 3935-3958

S 0002-9947(2011)05045-2

Article electronically published on March 10, 2011

\title{
EINSTEIN SOLVMANIFOLDS AND THE PRE-EINSTEIN DERIVATION
}

\author{
Y. NIKOLAYEVSKY
}

\begin{abstract}
An Einstein nilradical is a nilpotent Lie algebra which can be the nilradical of a metric Einstein solvable Lie algebra. The classification of Riemannian Einstein solvmanifolds (possibly, of all noncompact homogeneous Einstein spaces) can be reduced to determining which nilpotent Lie algebras are Einstein nilradicals and to finding, for every Einstein nilradical, its Einstein metric solvable extension. For every nilpotent Lie algebra, we construct an (essentially unique) derivation, the pre-Einstein derivation, the solvable extension by which may carry an Einstein inner product. Using the pre-Einstein derivation, we then give a variational characterization of Einstein nilradicals. As an application, we prove an easy-to-check convex geometry condition for a nilpotent Lie algebra with a nice basis to be an Einstein nilradical and also show that a typical two-step nilpotent Lie algebra is an Einstein nilradical.
\end{abstract}

\section{INTRODUCTION}

The theory of Riemannian homogeneous spaces with an Einstein metric splits into three very different cases depending on the sign of the Einstein constant, the scalar curvature. Among them, the picture is complete only in the Ricci-flat case: by the result of $\mathrm{AK}$, every Ricci-flat homogeneous space is flat.

The major open conjecture in the case of negative scalar curvature is the Alekseevski Conjecture Al1 asserting that a noncompact Einstein homogeneous space admits a simply transitive solvable isometry group. This is equivalent to saying that any such space is a solvmanifold, a solvable Lie group with a left-invariant Riemannian metric satisfying the Einstein condition.

By a deep result of J. Lauret La5, any Einstein solvmanifold is standard. This means that the metric solvable Lie algebra $\mathfrak{s}$ of such a solvmanifold has the following property: the orthogonal complement to the derived algebra of $\mathfrak{s}$ is abelian. The systematic study of standard Einstein solvmanifolds (and the term "standard") originated from the paper of J. Heber [Heb].

On the Lie algebra level, all the metric Einstein solvable Lie algebras can be obtained as the result of the following construction [Heb, La1, La5, LW]. One starts with the three pieces of data: a nilpotent Lie algebra $\mathfrak{n}$, a semisimple derivation $\Phi$ of $\mathfrak{n}$, and an inner product $\langle\cdot, \cdot\rangle_{\mathfrak{n}}$ on $\mathfrak{n}$, with respect to which $\Phi$ is symmetric. An extension of $\mathfrak{n}$ by $\Phi$ is a solvable Lie algebra $\mathfrak{s}=\mathbb{R} H \oplus \mathfrak{n}$ (as a linear space)

Received by the editors March 31, 2008 and, in revised form, March 10, 2009.

2000 Mathematics Subject Classification. Primary 53C30, 53C25.

Key words and phrases. Einstein solvmanifold, Einstein nilradical.

(C)2011 American Mathematical Society Reverts to public domain 28 years from publication 
with $\left(\operatorname{ad}_{H}\right)_{\mid \mathfrak{n}}:=\Phi$. The inner product on $\mathfrak{s}$ is defined by $\langle H, \mathfrak{n}\rangle=0$ and $\|H\|^{2}=$ $\operatorname{Tr} \Phi$ (and coincides with the existing one on $\mathfrak{n}$ ). The resulting metric solvable Lie algebra $(\mathfrak{s},\langle\cdot, \cdot\rangle)$ is Einstein provided $\mathfrak{n}$ is "nice" and the derivation $\Phi$ and the inner product $\langle\cdot, \cdot\rangle_{\mathfrak{n}}$ are chosen "in the correct way" (note, however, that these conditions are expressed by a system of algebraic equations, which could hardly be analyzed directly; see Section 2). Metric Einstein solvable Lie algebras of higher rank (with the codimension of the nilradical greater than one) having the same nilradical $\mathfrak{n}$ can be obtained from $\mathfrak{s}$ via a known procedure, by further adjoining to $\mathfrak{n}$ a semisimple derivation commuting with $\Phi$.

It turns out that the structure of an Einstein metric solvable Lie algebra is completely encoded in its nilradical in the following sense: given a nilpotent Lie algebra $\mathfrak{n}$, there is no more than one (possibly none) choice of $\Phi$ and of $\langle\cdot, \cdot\rangle_{\mathfrak{n}}$, up to conjugation by Aut(n) and scaling, which may result in an Einstein metric solvable Lie algebra $(\mathfrak{s},\langle\cdot, \cdot\rangle)$.

Definition 1. A nilpotent Lie algebra is called an Einstein nilradical if it is the nilradical of an Einstein metric solvable Lie algebra. A derivation $\Phi$ of an Einstein nilradical $\mathfrak{n}$ and an inner product $\langle\cdot, \cdot\rangle_{\mathfrak{n}}$, for which the metric solvable Lie algebra $(\mathfrak{s},\langle\cdot, \cdot\rangle)$ is Einstein, are called an Einstein derivation and a nilsoliton inner product, respectively.

In this paper, we address the following two questions:

(A) How do we determine whether a given nilpotent Lie algebra $\mathfrak{n}$ is an Einstein nilradical?

(B) If $\mathfrak{n}$ is an Einstein nilradical, how do we construct an Einstein solvmanifold whose Lie algebra has $\mathfrak{n}$ as its nilradical?

To answer question (B), we have to produce an Einstein derivation and a nilsoliton inner product for $\mathfrak{n}$. For an Einstein derivation, the answer is given by Theorem 1 below: any Einstein derivation is a positive multiple of a pre-Einstein derivation (which in practice can be found by solving a system of linear equations). The nilsoliton inner product could rarely be found explicitly, unless $\mathfrak{n}$ has a very simple structure. Implicitly it is characterized by (iii) of Theorem 2 and Remark 1 below (see also Theorem 5 in Section 3 ).

Question (A) is much more delicate. A necessary condition for a nilpotent Lie algebra to be an Einstein nilradical is that it admits an $\mathbb{N}$-gradation (which is defined by the Einstein derivation [Heb]). However, not every $\mathbb{N}$-graded nilpotent Lie algebra is an Einstein nilradical.

It is known, for instance, that the following nilpotent Lie algebras $\mathfrak{n}$ are Einstein nilradicals: $\mathfrak{n}$ is abelian Al2, $\mathfrak{n}$ has a codimension one abelian ideal La2, dim $\mathfrak{n} \leq 6$ Wil, La2, and $\mathfrak{n}$ is the algebra of strictly upper-triangular matrices Pay. Free Einstein nilradicals are classified in [Ni2]: apart from the abelian and the two-step ones, there are only six others. A characterization of the Einstein nilradical with a simple Einstein derivation and the classification of filiform Einstein nilradicals (modulo known classification of filiform $\mathbb{N}$-graded Lie algebras) is given in [Ni1].

Our starting point is the following fact: if $\Phi$ is an Einstein derivation of an Einstein nilradical $\mathfrak{n}$, then for some constant $c<0, \operatorname{Tr}(\Phi \psi)=-c \operatorname{Tr} \psi$, for any derivation $\psi$ of $\mathfrak{n}$ (see Section 2 for details). This motivates the following definition. 
Definition 2. A derivation $\phi$ of a Lie algebra $\mathfrak{n}$ is called pre-Einstein if it is semisimple, with all the eigenvalues real, and

$$
\operatorname{Tr}(\phi \psi)=\operatorname{Tr} \psi, \quad \text { for any } \psi \in \operatorname{Der}(\mathfrak{n}) .
$$

Here Der $(\mathfrak{n})$ is the algebra of derivations of $\mathfrak{n}$. We call an endomorphism $A$ of a linear space real (nonnegative, positive) if all its eigenvalues are real (respectively, nonnegative, positive). In the latter cases, we write $A \geq 0$ (respectively, $A>0$ ). For any $\psi \in \operatorname{Der}(\mathfrak{n})$ we denote by $\operatorname{ad}_{\psi}$ the corresponding inner derivation of $\operatorname{Der}(\mathfrak{n})$. If $\psi$ is semisimple and real, the same is true for $\operatorname{ad}_{\psi}$.

Our main result is contained in Theorems 1 and 2

\section{Theorem 1.}

1. (a) Any Lie algebra $\mathfrak{g}$ admits a pre-Einstein derivation $\phi_{\mathfrak{g}}$.

(b) The derivation $\phi_{\mathfrak{g}}$ is determined uniquely up to automorphism of $\mathfrak{g}$.

(c) All the eigenvalues of $\phi_{\mathfrak{g}}$ are rational numbers.

2. Let $\mathfrak{n}$ be a nilpotent Lie algebra with $\phi$ a pre-Einstein derivation. If $\mathfrak{n}$ is an Einstein nilradical, then its Einstein derivation is positively proportional to $\phi$, up to conjugation by $\operatorname{Aut}(\mathfrak{n})$, and

$$
\phi>0 \quad \text { and } \quad \operatorname{ad}_{\phi} \geq 0 .
$$

The inequalities (2) are necessary, but not sufficient to guarantee that a nilpotent Lie algebra is an Einstein nilradical. Combining the idea of the pre-Einstein derivation with [Heb, Theorem 6.15], we give a variational characterization of Einstein nilradicals, which answers question (A). Denote by $\mathcal{V}=\bigwedge^{2}\left(\mathbb{R}^{n}\right)^{*} \otimes \mathbb{R}^{n}$ the space of skew-symmetric bilinear maps on $\mathbb{R}^{n}$. Let $\mu$ be an element of $\mathcal{V}$ defining a nilpotent Lie algebra $\mathfrak{n}=\left(\mathbb{R}^{n}, \mu\right)$. Choose and fix a pre-Einstein derivation $\phi$ of $\mathfrak{n}$, and define the subalgebra $\mathfrak{g}_{\phi} \subset \mathfrak{s l}(\mathfrak{n})$ by

$$
\mathfrak{g}_{\phi}=\mathfrak{z}(\phi) \cap \operatorname{Ker}(t),
$$

where $\mathfrak{z}(\phi)$ is the centralizer of $\phi$ in $\mathfrak{s l}(n)$ and $t$ is a linear functional on $\mathfrak{s l}(n)$ defined by $t(A)=\operatorname{Tr}(A \phi)$. Let $G_{\phi} \subset \mathrm{SL}(n)$ be the connected Lie group with the Lie algebra $\mathfrak{g}_{\phi}$ (explicitly given by (9)). Define the action of $G_{\phi}$ on the linear space $\mathcal{V}$ by $g . \nu(X, Y)=g \nu\left(g^{-1} X, g^{-1} Y\right)$ for $\nu \in \mathcal{V}, g \in G_{\phi}$.

Choose an arbitrary inner product $\langle\cdot, \cdot\rangle$ on $\mathbb{R}^{n}$, with respect to which $\phi$ is symmetric, and define $\|\nu\|^{2}=\sum_{i, j}\left\|\nu\left(E_{i}, E_{j}\right)\right\|^{2}$, where $\left\{E_{i}\right\}$ is an orthonormal basis for $\mathbb{R}^{n}$ and $\nu \in \mathcal{V}$.

Theorem 2. For a nilpotent Lie algebra $\mathfrak{n}=\left(\mathbb{R}^{n}, \mu\right)$ with a pre-Einstein derivation $\phi$, the following conditions are equivalent:

(i) $\mathfrak{n}$ is an Einstein nilradical;

(ii) the orbit $G_{\phi} \cdot \mu \subset \mathcal{V}$ is closed;

(iii) the function $f: G_{\phi} \rightarrow \mathbb{R}^{n}$ defined by $f(g)=\|g \cdot \mu\|^{2}$ has a critical point.

Remark 1. Note that the equivalence of (i) and (ii) gives the characterization of Einstein nilradicals completely in terms of the Lie algebra structure of $\mathfrak{n}$, with no inner product involved.

The equivalence of (i) and (iii) is similar to the well-known criterion for a homogeneous space of a unimodular Lie group to be Einstein: as it follows from (4) of Section 22 the scalar curvature of a metric nilpotent Lie algebra is the squared norm of the Lie bracket times a constant. 
In (iii), one can replace "has a critical point" by "attains the minimum". Moreover, if $f$ attains the minimum at $g \in G_{\phi}$ and $\mu_{0}=g . \mu$, then the metric Lie algebra $\left(\mathbb{R}^{n}, \mu_{0},\langle\cdot, \cdot\rangle\right)$ is isometrically isomorphic to the nilradical of an Einstein metric solvable Lie algebra (see Theorem 5 in Section 3 ).

Note in conjunction with (ii) that the $\mathrm{SL}(n)$-orbit of a nonabelian nilpotent Lie algebra is never closed [La3, Theorem 8.2]. One can therefore either work with a smaller group (as in Theorem 21) or consider a different function. One such function naturally related to the group action is the squared norm of the moment map. As shown in [La4, LW], a nilpotent Lie algebra $\mathfrak{n}=\left(\mathbb{R}^{n}, \mu\right)$ is an Einstein nilradical if and only if the squared norm of the moment map (with respect to some inner product on $\mathbb{R}^{n}$ ) of the $\mathrm{SL}(n)$-action on $\mathcal{V}$ attains its minimum on the orbit of $\mu$.

As an application of Theorems 1 and 2, we consider nilpotent Lie algebras having a nice basis.

Definition 3. Let $\left\{X_{1}, \ldots, X_{n}\right\}$ be a basis for a nilpotent Lie algebra $\mathfrak{n}$, with $\left[X_{i}, X_{j}\right]=\sum_{k} c_{i j}^{k} X_{k}$. The basis $\left\{X_{i}\right\}$ is called nice if for every $i, j, \#\left\{k: c_{i j}^{k} \neq 0\right\}$ $\leq 1$, and for every $i, k, \#\left\{j: c_{i j}^{k} \neq 0\right\} \leq 1$.

Although the condition of having a nice basis looks rather restrictive (some examples of nilpotent Lie algebras having no nice basis are given in Section 44), the nilpotent Lie algebras with a nice basis are not uncommon. Such algebras often appear in the classification lists, especially in the low-dimensional cases. Every Lie algebra admitting a derivation with all the eigenvalues of multiplicity one has a nice basis (recall that to be an Einstein nilradical, a nilpotent Lie algebra must admit a positive real semisimple derivation). In particular, filiform algebras admitting an $\mathbb{N}$-gradation have a nice basis [Ni1. Every two-step nilpotent algebra attached to a graph has a nice basis $[\mathrm{LW}$.

Given a nilpotent algebra $\mathfrak{n}$ of dimension $n$ with a nice basis, introduce the following objects. In a Euclidean space $\mathbb{R}^{n}$ with the inner product $(\cdot, \cdot)$ and an orthonormal basis $f_{1}, \ldots, f_{n}$, define the finite subset $\mathbf{F}=\left\{(Y)_{i j}^{k}=f_{i}+f_{j}-f_{k}\right.$ : $\left.c_{i j}^{k} \neq 0, i<j\right\}$. Denote by $L$ the affine span of $\mathbf{F}$, the smallest affine subspace of $\mathbb{R}^{n}$ containing $\mathbf{F}$, and $\operatorname{Conv}(\mathbf{F})$ the convex hull of $\mathbf{F}$. Let $m=\# \mathbf{F}$. Fix an arbitrary ordering of the set $\mathbf{F}$ and define an $m \times n$ matrix $Y$ with the rows $(Y)_{i j}^{k}$. Namely, if for $1 \leq a \leq m$ the $a$-th element of $\mathbf{F}$ is $f_{i}+f_{j}-f_{k}$, then the $a$-th row of $Y$ has 1 in the columns $i$ and $j,-1$ in the column $k$, and zero elsewhere. Denote by $[1]_{m}$ an $m$-dimensional vector all of whose coordinates are ones.

We have the following theorem:

Theorem 3. A nonabelian nilpotent Lie algebra $\mathfrak{n}$ with a nice basis is an Einstein nilradical if and only if any of the following two equivalent conditions hold:

(i) The projection of the origin of $\mathbb{R}^{n}$ to $L$ lies in the interior of $\operatorname{Conv}(\mathbf{F})$.

(ii) There exists a vector $\alpha \in \mathbb{R}^{m}$ with positive coordinates satisfying $Y Y^{t} \alpha=$ $[1]_{m}$.

Note that by [Pay, Theorem 1], a metric nilpotent Lie algebra is nilsoliton if and only if the equation $Y Y^{t} \alpha=-2 c[1]_{m}$ holds with respect to the basis of Ricci eigenvectors (where the components of $\alpha$ are the squares of the structural constants).

As another application of Theorems 1 and 2, we prove Theorem 6, which says that a nilpotent Lie algebra, which is complex isomorphic to an Einstein nilradical, is an Einstein nilradical by itself, and Theorem 7 , which shows that the direct sum 
of nilpotent Lie algebras is an Einstein nilradical if and only if all the summands are Einstein nilradicals (see Section 4).

In Section 5, we consider two-step nilpotent Einstein nilradicals. Informally, we prove that

a typical two-step nilpotent Lie algebra is an Einstein nilradical of eigenvalue type $(1,2 ; q, p)$.

There seems to be no commonly accepted notion as to what is a "typical" nilpotent Lie algebra [Luk. We mean the following. A two-step nilpotent Lie algebra $\mathfrak{n}$ is said to be of type $(p, q)$ if $\operatorname{dim} \mathfrak{n}=p+q$ and $\operatorname{dim}[\mathfrak{n}, \mathfrak{n}]=p\left(\right.$ clearly, $\left.1 \leq p \leq \frac{1}{2} q(q-1)\right)$. Any such algebra is determined by a point in the linear space $\mathcal{V}(p, q)=\left(\bigwedge^{2} \mathbb{R}^{q}\right)^{p}$, with two points giving isomorphic algebras if and only if they lie on the same orbit of the action of $\operatorname{GL}(q) \times \mathrm{GL}(p)$ on $\mathcal{V}(p, q)$. The space of isomorphism classes of the algebras of type $(p, q)$ is a compact non-Hausdorff space, the quotient of an open and dense subset of $\mathcal{V}(p, q)$ by the action of $\operatorname{GL}(q) \times \mathrm{GL}(p)$.

Theorem 4. Suppose $q \geq 6$ and $2<p<\frac{1}{2} q(q-1)-2$, or $(p, q)=(5,5)$. Then:

(i) there are continuum isomorphism classes of two-step nilpotent Lie algebras of type $(p, q)$; each of them has an empty interior in the space $\mathcal{V}(p, q)$ [Eb1;

(ii) the space $\mathcal{V}(p, q)$ contains an open and dense subset corresponding to twostep nilpotent Einstein nilradicals of eigenvalue type $(1,2 ; q, p) \mathbb{1}$

Two-step nilpotent Lie algebras of the types excluded by Theorem 4 can be completely classified [GT. Using Theorem 3 we find all the two-step nilpotent Einstein nilradicals with $q \leq 5,(p, q) \neq(5,5)$ (up to complexification).

The paper is organized as follows. Section 2 gives the background on Einstein solvmanifolds. In Section 3 , we prove Theorem 1 and Theorem 5 which implies Theorem 2, and also give a possible general strategy of determining whether a given nilpotent Lie algebra is an Einstein nilradical. Section 4 contains three applications of Theorem 1 and Theorem 2. Theorem 3 on Einstein nilradicals with a nice basis, Theorem 6, which shows that the property of being an Einstein nilradical is a property of the complexification of a real nilpotent Lie algebra, and Theorem 7 on the direct sum of Einstein nilradicals. In Section 5 , we prove Theorem 4 and classify low-dimensional two-step Einstein nilradicals (Proposition 2).

The author would like to thank the referee for useful comments and suggestions.

\section{EINSTEIN SOLVMANIFOLDS}

Let $G$ be a Lie group with a left-invariant metric $Q$ obtained by the left translations from an inner product $\langle\cdot, \cdot\rangle$ on the Lie algebra $\mathfrak{g}$ of $G$. Let $B$ be the Killing form of $\mathfrak{g}$, and let $H \in \mathfrak{g}$ be the mean curvature vector defined by $\langle H, X\rangle=\operatorname{Tr} \operatorname{ad}_{X}$.

The Ricci curvature ric of the metric Lie group $(G, Q)$ at the identity is given by

$$
\operatorname{ric}(X)=-\langle[H, X], X\rangle-\frac{1}{2} B(X, X)-\frac{1}{2} \sum_{i}\left\|\left[X, E_{i}\right]\right\|^{2}+\frac{1}{4} \sum_{i, j}\left\langle\left[E_{i}, E_{j}\right], X\right\rangle^{2},
$$

for $X \in \mathfrak{g}$, where $\left\{E_{i}\right\}$ is an orthonormal basis for $(\mathfrak{g},\langle\cdot, \cdot\rangle)$ [Heb, Eq. (2.3)].

\footnotetext{
${ }^{1}$ After this paper was written, the author became aware that the result equivalent to Theorem 4 is independently proved in Eb3.
} 
Equivalently, one can define the Ricci operator Ric of the metric Lie algebra $(\mathfrak{g},\langle\cdot, \cdot\rangle)$ (the symmetric operator associated to ric) by

$\operatorname{Tr}\left(\left(\operatorname{Ric}+\frac{1}{2}\left(\operatorname{ad}_{H}+\operatorname{ad}_{H}^{*}\right)+\frac{1}{2} B\right) A\right)=\frac{1}{4} \sum_{i, j}\left\langle A\left[E_{i}, E_{j}\right]-\left[A E_{i}, E_{j}\right]-\left[E_{i}, A E_{j}\right],\left[E_{i}, E_{j}\right]\right\rangle$,

for any $A \in \operatorname{End}(\mathfrak{g})\left(\right.$ where $\operatorname{ad}_{H}^{*}$ is the metric adjoint of $\left.\operatorname{ad}_{H}\right)$.

If $(\mathfrak{n},\langle\cdot, \cdot\rangle)$ is a nilpotent metric Lie algebra, then $H=0$ and $B=0$, so

$$
\operatorname{Tr}\left(\operatorname{Ric}_{\mathfrak{n}} A\right)=\frac{1}{4} \sum_{i, j}\left\langle A\left[E_{i}, E_{j}\right]-\left[A E_{i}, E_{j}\right]-\left[E_{i}, A E_{j}\right],\left[E_{i}, E_{j}\right]\right\rangle,
$$

for any $A \in \operatorname{End}(\mathfrak{n})$. Explicitly, for $X, Y \in \mathfrak{n}$,

$$
\left.\left\langle\operatorname{Ric}_{\mathfrak{n}} X, Y\right\rangle=\frac{1}{4} \sum_{i, j}\left\langle X,\left[E_{i}, E_{j}\right]\right\rangle\left\langle Y,\left[E_{i}, E_{j}\right]\right\rangle-\frac{1}{2} \sum_{i, j}\left\langle\left[X, E_{i}\right], E_{j}\right\rangle\left\langle\left[Y, E_{i}\right], E_{j}\right]\right\rangle .
$$

By the result of [La5], any Einstein metric solvable Lie algebra is standard, which means that the orthogonal complement to the derived algebra $[\mathfrak{g}, \mathfrak{g}]$ is abelian.

It is proved in $\mathrm{AK}$ that any Ricci-flat metric solvable Lie algebra is flat. By [DM], any Einstein metric solvable unimodular Lie algebra is also flat. We will therefore always assume $\mathfrak{g}$ to be nonunimodular $(H \neq 0)$, with an inner product of strictly negative scalar curvature $c \operatorname{dim} \mathfrak{g}$.

Any Einstein metric solvable Lie algebra admits a rank-one reduction Heb, Theorem 4.18]. This means that if $(\mathfrak{g},\langle\cdot, \cdot\rangle)$ is such an algebra, with the nilradical $\mathfrak{n}$ and the mean curvature vector $H$, then the subalgebra $\mathfrak{g}_{1}=\mathbb{R} H \oplus \mathfrak{n}$, with the induced inner product, is also Einstein. What is more, the derivation $\Phi=\operatorname{ad}_{H \mid \mathfrak{n}}: \mathfrak{n} \rightarrow \mathfrak{n}$ can be assumed symmetric up to isometry, and all its eigenvalues belong to $\gamma \mathbb{N}$ for some $\gamma>0$. This implies, in particular, that the nilradical $\mathfrak{n}$ of an Einstein metric solvable Lie algebra admits an $\mathbb{N}$-gradation defined by the eigenspaces of $\Phi$. By [La1, Theorem 3.7], a necessary and sufficient condition for a metric nilpotent algebra $(\mathfrak{n},\langle\cdot, \cdot\rangle)$ to be the nilradical of an Einstein metric solvable Lie algebra is

$$
\operatorname{Ric}_{\mathfrak{n}}=c \operatorname{id}_{\mathfrak{n}}+\Phi, \quad \text { for some } \Phi \in \operatorname{Der}(\mathfrak{n}),
$$

where $c \operatorname{dim} \mathfrak{g}<0$ is the scalar curvature of $(\mathfrak{g},\langle\cdot, \cdot\rangle)$. This equation, in fact, defines $(\mathfrak{g},\langle\cdot, \cdot\rangle)$ in the following sense: given a metric nilpotent Lie algebra whose Ricci operator satisfies ([6), with some constant $c<0$ and some $\Phi \in \operatorname{Der}(\mathfrak{n})$, one can define $\mathfrak{g}$ as a one-dimensional extension of $\mathfrak{n}$ by $\Phi$. For such an extension $\mathfrak{g}=$ $\mathbb{R} H \oplus \mathfrak{n}, \operatorname{ad}_{H \mid \mathfrak{n}}=\Phi$, and the inner product defined by $\langle H, \mathfrak{n}\rangle=0,\|H\|^{2}=\operatorname{Tr} \Phi$ (and coinciding with the existing one on $\mathfrak{n}$ ) is Einstein, with scalar curvature $c \operatorname{dim} \mathfrak{g}$. A nilpotent Lie algebra $\mathfrak{n}$ which admits an inner product $\langle\cdot, \cdot\rangle$ and a derivation $\Phi$ satisfying (6) is called an Einstein nilradical, the corresponding derivation $\Phi$ is called an Einstein derivation, and the inner product $\langle\cdot, \cdot\rangle$ the nilsoliton metric.

As proved in [La1, Theorem 3.5], a nilpotent Lie algebra admits no more than one nilsoliton metric, up to conjugation by Aut(n) and scaling (and hence, an Einstein derivation, if it exists, is unique, up to conjugation and scaling). Equation (6), together with (4), implies that if $\mathfrak{n}$ is an Einstein nilradical, with $\Phi$ the Einstein derivation, then for some $c<0$

$$
\operatorname{Tr}(\Phi \psi)=-c \operatorname{Tr} \psi, \quad \text { for any } \psi \in \operatorname{Der}(\mathfrak{n}) .
$$

The set of eigenvalues $\lambda_{i}$ and their multiplicities $d_{i}$ of the Einstein derivation $\Phi$ of an Einstein nilradical $\mathfrak{n}$ is called the eigenvalue type of $\mathfrak{n}$ (and of $\Phi$ ). The eigenvalue 
type is usually written as $\left(\lambda_{1}, \ldots, \lambda_{p} ; d_{1}, \ldots, d_{p}\right)$ (note that the $\lambda_{i}$ 's are defined up to positive multiple).

Throughout the paper, $\oplus$ means the direct sum of linear spaces (even when the summands are Lie algebras). Any semisimple endomorphism $A$ of a linear space $V$ admits a decomposition into the real and the imaginary part: $A=A^{\mathbb{R}}+A^{i \mathbb{R}}$. The operator $A^{\mathbb{R}}$ is defined as follows: if $V_{1}, \ldots, V_{m}$ are the eigenspaces of $A$ acting on $V^{\mathbb{C}}$, with eigenvalues $a_{1}, \ldots, a_{m} \in \mathbb{C}$, respectively, then $A^{\mathbb{R}}$ acts by multiplication by $b \in \mathbb{R}$ on every subspace $\left(\bigoplus_{k: \operatorname{Re} a_{k}=b} V_{k}\right) \cap V$. For any semisimple $A \in \operatorname{End}(V)$, the operators $A, A^{\mathbb{R}}$, and $A^{i \mathbb{R}}$ commute. If $\psi$ is a semisimple derivation of a Lie algebra $\mathfrak{g}$, then both $\psi^{\mathbb{R}}$ and $\psi^{i \mathbb{R}}$ are also derivations.

\section{Pre-Einstein derivation. Proof of Theorems 1 and 2}

In this section, we prove Theorem 1 and Theorem 5 which contains Theorem 2 , and describe a possible general approach to questions (A) and (B) from Section 1 .

Proof of Theorem 1. 1. (a) The algebra $\operatorname{Der}(\mathfrak{g})$ is algebraic. Let $\operatorname{Der}(\mathfrak{g})=\mathfrak{s} \oplus \mathfrak{t} \oplus \mathfrak{n}$ be its Levi-Mal'cev decomposition, where $\mathfrak{t} \oplus \mathfrak{n}$ is the radical of $\operatorname{Der}(\mathfrak{g}), \mathfrak{s}$ is semisimple, $\mathfrak{n}$ is the set of all nilpotent elements in $\mathfrak{t} \oplus \mathfrak{n}$ (and is the nilradical of $\mathfrak{t} \oplus \mathfrak{n}$ ), $\mathfrak{t}$ is a torus, an abelian subalgebra consisting of semisimple elements, and $[\mathfrak{t}, \mathfrak{s}]=0$. With any $\psi \in \mathfrak{t}, \psi^{\mathbb{R}}$ and $\psi^{i \mathbb{R}}$ are also in $\mathfrak{t}$. The subspaces $\mathfrak{t}_{c}=\left\{\psi^{i \mathbb{R}}: \psi \in \mathfrak{t}\right\}$ and $\mathfrak{t}_{s}=\left\{\psi^{\mathbb{R}}: \psi \in \mathfrak{t}\right\}$ are the compact and the fully $\mathbb{R}$-reducible tori (the elements of $\mathfrak{t}_{s}$ are diagonal matrices in some basis for $\left.\mathfrak{g}\right), \mathfrak{t}_{s} \oplus \mathfrak{t}_{c}=\mathfrak{t}$.

The quadratic form $b$ defined on $\operatorname{Der}(\mathfrak{g})$ by $b\left(\psi_{1}, \psi_{2}\right)=\operatorname{Tr}\left(\psi_{1} \psi_{2}\right)$ is invariant $\left(b\left(\psi_{1},\left[\psi_{2}, \psi_{3}\right]\right)=b\left(\left[\psi_{1}, \psi_{3}\right], \psi_{2}\right)\right)$. In general, $b$ is degenerate, with Ker $b=\mathfrak{n}$, so for any $\psi \in \mathfrak{n}, b(\mathfrak{t}, \psi)=\operatorname{Tr} \psi=0$. As $\mathfrak{s}$ is semisimple and $[\mathfrak{t}, \mathfrak{s}]=0$, we also have $b(\mathfrak{t}, \psi)=\operatorname{Tr} \psi=0$, for any $\psi \in \mathfrak{s}$. Moreover, for any $\psi \in \mathfrak{t}_{c}, b\left(\mathfrak{t}_{s}, \psi\right)=\operatorname{Tr} \psi=0$.

Hence to find a pre-Einstein derivation for $\mathfrak{g}$ it suffices to find an element $\phi \in \mathfrak{t}_{s}$ which satisfies (11), for all $\psi \in \mathfrak{t}_{s}$. Such a $\phi$ indeed exists, as the restriction of $b$ to $\mathfrak{t}_{s}$ is nondegenerate (even definite) and is unique, when a particular torus $\mathfrak{t}$ is chosen.

(b) The subalgebra $\mathfrak{s} \oplus \mathfrak{t}$ is a maximal fully reducible subalgebra of $\operatorname{Der}(\mathfrak{g})$. As by Mos, Theorem 4.1], the maximal fully reducible subalgebras of $\operatorname{Der}(\mathfrak{g})$ are conjugate by an inner automorphism of $\operatorname{Der}(\mathfrak{g})$ (which corresponds to an automorphism of $\mathfrak{g}$ ), and then $\mathfrak{t}$, the center of $\mathfrak{s} \oplus \mathfrak{t}$, is defined uniquely, and the uniqueness of $\phi$, up to automorphism, follows.

(c) The proof is similar to that of [Heb, Theorem 4.14]. Suppose $\phi$ has eigenvalues $\lambda_{i}$, with multiplicities $d_{i}$, respectively, $i=1, \ldots, p$. In a Euclidean space $\mathbb{R}^{p}$ with a fixed orthonormal basis $f_{i}$, consider all the vectors of the form $f_{i}+f_{j}-f_{k}$ such that $\lambda_{i}+\lambda_{j}-\lambda_{k}=0$. In their linear span choose a basis $v_{k}, k=1, \ldots, m$, consisting of vectors of the above form and introduce a $p \times m$ matrix $F$ whose vector-columns are the $v_{k}$ 's. Then any vector $\nu=\left(\nu_{1}, \ldots, \nu_{p}\right)^{t} \in \mathbb{R}^{p}$ satisfying $F^{t} \nu=0$ defines a derivation $\psi=\psi(\nu)$ having the same eigenspaces as $\phi$, but with the corresponding eigenvalues $\nu_{i}$. From (1) we must have $\sum d_{i}\left(\lambda_{i}-1\right) \nu_{i}=0$, for any such $\nu$, which implies that the vector $\left(d_{1}\left(\lambda_{1}-1\right), \ldots, d_{p}\left(\lambda_{p}-1\right)\right)^{t}$ belongs to the column space of $F$. So there exists $x \in \mathbb{R}^{m}$ such that $\lambda=[1]_{p}+D^{-1} F x$, where $\lambda=\left(\lambda_{1}, \ldots, \lambda_{p}\right)^{t},[1]_{p}=$ $(1, \ldots, 1)^{t} \in \mathbb{R}^{p}$, and $D=\operatorname{diag}\left(d_{1}, \ldots, d_{p}\right)$. As $\phi$ by itself is a derivation, we have $F^{t} \lambda=0$, which implies $F^{t}[1]_{p}+F^{t} D^{-1} F x=0$, so that $x=-\left(F^{t} D^{-1} F\right)^{-1}[1]_{m}$, as $F^{t}[1]_{p}=[1]_{m}$ and $\mathrm{rk} F=m$. Then $\lambda=[1]_{p}-D^{-1} F\left(F^{t} D^{-1} F\right)^{-1}[1]_{m}$ and the claim follows, as all the entries of $D$ and of $F$ are integers. 
2. Suppose that $\mathfrak{n}$ is an Einstein nilradical, with an Einstein derivation $\Phi$ and a nilsoliton inner product $\langle\cdot, \cdot\rangle$. Then $\Phi$ is semisimple, real and satisfies (7), with some $c<0$, so $\phi=(-c)^{-1} \Phi$ is a pre-Einstein derivation. Moreover, $\Phi>0$ (as follows from [Heb, Theorem 4.14]) and $\operatorname{ad}_{\Phi} \geq 0$. To prove the latter inequality, we use the fact that for any $\psi \in \operatorname{Der}(\mathfrak{n}), \operatorname{Tr}\left(\Phi\left[\psi, \psi^{*}\right]\right) \geq 0$ by [Nik, Corollary 5] and (6). If $\operatorname{ad}_{\Phi} \psi=\lambda \psi$ for some $\lambda \in \mathbb{R}$, then $\lambda \operatorname{Tr}\left(\psi \psi^{*}\right)=\operatorname{Tr}\left([\Phi, \psi] \psi^{*}\right)=\operatorname{Tr}\left(\Phi\left[\psi, \psi^{*}\right]\right) \geq$ 0 .

Note that the pre-Einstein derivation of a semisimple Lie algebra is trivial (zero), and it may well happen that the pre-Einstein derivation of a nilpotent Lie algebra is zero (for instance, for a characteristically nilpotent algebra).

Inequalities (2) from assertion 2 of Theorem 1 can be used to prove that certain nilpotent Lie algebras are not Einstein nilradicals. Of course, if $\mathfrak{n}$ has no positive derivations at all, it is not an Einstein nilradical. Nilpotent algebras whose preEinstein derivation is positive, but the inequality $\operatorname{ad}_{\phi} \geq 0$ is violated, are more common. One example is given below.

Example 1. Let $\mathfrak{n}$ be a two-step nilpotent 12-dimensional algebra of type $(2,10)$ (see Section 5) defined by the relations $\left[X_{1}, X_{3}\right]=\left[X_{2}, X_{4}\right]=\left[X_{5}, X_{9}\right]=\left[X_{6}, X_{10}\right]$ $=Z_{1}$ and $\left[X_{1}, X_{4}\right]=\left[X_{5}, X_{8}\right]=\left[X_{6}, X_{9}\right]=\left[X_{7}, X_{10}\right]=Z_{2}$. A direct computation (based, for instance, on [Luk, Proposition 3.1]) shows that the derivation $\phi$ given by the diagonal matrix $\frac{1}{55} \operatorname{diag}(43,42,42,43,42,43,44,44,43,42,85,86)$ with respect to the basis $\left\{X_{i}, Z_{\alpha}\right\}$ is pre-Einstein. An endomorphism $\psi$ sending $X_{4}$ to $X_{2}$ and all the other basis vectors to zero is a derivation satisfying $\operatorname{ad}_{\phi} \psi=-\frac{1}{55} \psi$.

In the proof of Theorem 2, we combine the idea of the pre-Einstein derivation with [Heb, Theorem 6.15], which in turn uses the results of [RS] (see also La4, Section 6]). The group $G_{\phi}$ is explicitly constructed as follows. Given a nilpotent algebra $\mathfrak{n}$, fix a pre-Einstein derivation $\phi$. Let $\mathfrak{n}_{j}$ be the eigenspaces of $\phi$, with the corresponding eigenvalues $\lambda_{j}, j=1, \ldots, p$. Denote $a_{j}=N \lambda_{j}$, where $N$ is the least common multiple of the denominators of the $\lambda_{j}$ 's. Then $G_{\phi}$ is the identity component of the subgroup $\tilde{G}_{\phi} \subset \prod_{j=1}^{p} \mathrm{GL}\left(\mathfrak{n}_{j}\right) \subset \mathrm{GL}(n)$ defined by

$$
\tilde{G}_{\phi}=\left\{\left(g_{1}, \ldots, g_{p}\right): g_{j} \in \mathrm{GL}\left(\mathfrak{n}_{j}\right), \prod_{j=1}^{p} \operatorname{det} g_{j}=\prod_{j=1}^{p}\left(\operatorname{det} g_{j}\right)^{a_{j}}=1\right\} .
$$

As all the $a_{j}$ are integers (although some could be zero or negative), the group $\tilde{G}_{\phi}$ is real algebraic. The group $G_{\phi}$ is reductive, with the Lie algebra $\mathfrak{g}_{\phi}$, and is given explicitly by

$$
G_{\phi}=\tilde{G}_{\phi} \cap \prod_{j=1}^{p} \mathrm{GL}^{+}\left(\mathfrak{n}_{j}\right), \quad \text { where } \mathrm{GL}^{+}(V)=\{g \in \mathrm{GL}(V): \operatorname{det} g>0\} .
$$

We start with the following technical lemma (cf. [Heb, Section 6.4]).

Lemma 1. $\quad$ 1. Let $\mathfrak{n}=\left(\mathbb{R}^{n}, \mu\right)$ be a nilpotent Lie algebra with a pre-Einstein derivation $\phi$. Then $\phi$ is a pre-Einstein derivation for every algebra $\left(\mathbb{R}^{n}, g . \mu\right)$, $g \in G_{\phi}$. The group $G_{\phi}$ and the orbit $G_{\phi} \cdot(g . \mu)$ are the same, for all $g \in G_{\phi}$.

2. Let $\mathfrak{n}=\left(\mathbb{R}^{n}, \mu\right)$ be a nilpotent Lie algebra and let $\psi$ be a semisimple derivation of $\mathfrak{n}$ with rational eigenvalues (not necessarily a pre-Einstein). Let $\langle\cdot, \cdot\rangle$ be an inner product on $\mathfrak{n}$, with respect to which $\psi$ is symmetric. Define the algebra $\mathfrak{g}_{\psi}$ and the group $G_{\psi}$ for $\psi$ by (3) and (9), respectively, and the function $f: G_{\psi} \rightarrow \mathbb{R}$ by $f(g)=\|g \cdot \mu\|^{2}$. Then:

(a) For every $g \in G_{\psi}$ and $A \in \operatorname{End}(\mathfrak{n}), \operatorname{Tr}\left(\operatorname{Ric}_{g \cdot \mu} A\right)=\left.\frac{1}{8} \frac{d}{d t}\right|_{t=0} f(\exp (t A) g)$. 
(b) Let $Q$ be an inner product on $\operatorname{End}(\mathfrak{n})$ defined by $Q\left(A_{1}, A_{2}\right)=\operatorname{Tr}\left(A_{1} A_{2}^{*}\right)$ (adjoint with respect to $\langle\cdot, \cdot\rangle$ ), and $\mathfrak{Z}(\psi)$ be the centralizer of $\psi$ in $\operatorname{End}(\mathfrak{n})$. Then for every $g \in G_{\psi}$ and $A \perp \mathfrak{Z}(\psi),\left.\frac{d}{d t}\right|_{t=0} f(\exp (t A) g)=0$.

3. In the settings of $\mathbb{1}$, suppose that the function $f$ has a critical point $g_{0} \in G_{\psi}$. Then:

(a) $\mathfrak{n}$ is an Einstein nilradical, with $\langle\cdot, \cdot\rangle$ a nilsoliton metric for $\left(\mathbb{R}^{n}, g_{0} \cdot \mu\right)$.

(b) If $\mathfrak{n}$ is nonabelian, then $\psi$ is proportional to a pre-Einstein derivation of $\mathfrak{n}$.

Proof. 1. It is easy to see that $\operatorname{Der}(g \cdot \mu)=g \operatorname{Der}(\mu) g^{-1}$, for any $g \in \operatorname{GL}(n)$. In particular, if $g \in G_{\phi}$ (and hence commutes with $\phi$ ), the endomorphism $\phi$ is a real semisimple derivation of $\left(\mathbb{R}^{n}, g . \mu\right)$ satisfying (10) with any $\psi \in \operatorname{Der}(g . \mu)$.

2. Assertion (a) follows from (4) (with the Lie bracket $[\cdot, \cdot]=g \cdot \mu$ ). Assertion (b) follows from (a) and the fact that the Ricci tensor commutes with every symmetric derivation [Heb, Lemma 2.2], in particular, with $\psi$.

3. From the assumption and assertion 1 we obtain that $\operatorname{Tr}\left(\operatorname{Ric}_{g_{0} . \mu} A\right)=0$, for all $A \in \mathfrak{g}_{\psi} \oplus(\mathfrak{Z}(\psi))^{\perp}$. It follows from (3) that $\operatorname{Ric}_{g_{0} . \mu} \in \operatorname{Span}(\mathrm{id}, \psi)$; that is, equation (6) holds, with some $c \in \mathbb{R}$ and some $\psi \in \operatorname{Der}\left(g_{0} \cdot \mu\right)$. Then $\langle\cdot, \cdot\rangle$ is a nilsoliton metric for $\left(\mathbb{R}^{n}, g_{0} \cdot \mu\right)$ by [La1, Theorem 3.7]. The nilsoliton inner product for $\mathfrak{n}=\left(\mathbb{R}^{n}, \mu\right)$ can then be taken as $\langle X, Y\rangle^{\prime}=\left\langle g_{0}^{-1} X, g_{0}^{-1} Y\right\rangle$, for $X, Y \in \mathbb{R}^{n}$. This proves (a). To prove (b), note that $\operatorname{Ric}_{g_{0} . \mu}=c$ id $+a \psi$ for some $c, a \in \mathbb{R}$, as shown above, and $\operatorname{Ric}_{g_{0} . \mu}=c_{1}$ id $+\Phi$, for some $c_{1} \in \mathbb{R}$, where $\Phi$ is the Einstein derivation of the metric Lie algebra $\left(\mathbb{R}^{n}, g_{0} \cdot \mu,\langle\cdot, \cdot\rangle\right)$. As by (4), $\operatorname{Tr}\left(\operatorname{Ric}_{g_{0} . \mu} \psi\right)=\operatorname{Tr}\left(\operatorname{Ric}_{g_{0} . \mu} \Phi\right)=0$ and $\operatorname{Tr} \operatorname{Ric}_{g_{0} . \mu}<0$ when $\mathfrak{n}$ is nonabelian, and $c=c_{1}=\operatorname{Tr}\left(\operatorname{Ric}_{g_{0} . \mu}^{2}\right) / \operatorname{Tr}\left(\operatorname{Ric}_{g_{0} . \mu}\right)$, so $\Phi=a \psi$. By assertion 2 of Theorem 1 $\Phi=a_{1} \phi$ for some $a_{1} \in \mathbb{R}$, where $\phi$ is a pre-Einstein derivation for $\left(\mathbb{R}^{n}, g_{0} \cdot \mu\right)$, and hence for $\mathfrak{n}$ by assertion 1 . If $a=0$, then $\operatorname{Ric}_{g_{0} . \mu}=c$ id, and $\mathfrak{n}$ is again abelian by [Mil, Theorem 2.4], so $\psi=a^{-1} a_{1} \phi$.

Using the results of [RS] and Lemma 1 we prove the following theorem, which contains Theorem 2

Theorem 5. Let $\mathfrak{n}=\left(\mathbb{R}^{n}, \mu\right)$ be a nilpotent Lie algebra with a pre-Einstein derivation $\phi$. Let $\langle\cdot, \cdot\rangle$ be an inner product on $\mathfrak{n}$, with respect to which $\phi$ is symmetric. Define the algebra $\mathfrak{g}_{\phi}$ and the group $G_{\phi}$ by (3) and (9), respectively, and the function $f: G_{\phi} \rightarrow \mathbb{R}$ by $f(g)=\|g \cdot \mu\|^{2}$.

1. The following conditions are equivalent:

(i) $\mathfrak{n}$ is an Einstein nilradical;

(ii) the orbit $G_{\phi} \cdot \mu \subset \mathcal{V}$ is closed;

(iii) the function $f: G_{\phi} \rightarrow \mathbb{R}^{n}$ has a critical point;

(iv) the function $f: G_{\phi} \rightarrow \mathbb{R}^{n}$ attains its minimum.

If $g_{0} \in G_{\phi}$ is a critical point for $f$, then $\left\langle g_{0} \cdot, g_{0} \cdot\right\rangle$ is a nilsoliton inner product for $\mathfrak{n}$.

2. Suppose the orbit $G_{\phi} \cdot \mu$ is not closed. Then there exists a unique closed orbit $G_{\phi} \cdot \mu_{0} \subset \overline{G_{\phi} \cdot \mu}$. The following holds:

(a) the algebra $\mathfrak{n}_{0}=\left(\mathbb{R}^{n}, \mu_{0}\right)$ is an Einstein nilradical not isomorphic to $\mathfrak{n}$;

(b) either $\phi$ is a pre-Einstein derivation for $\mathfrak{n}_{0}$ or $\mathfrak{n}_{0}$ is abelian;

(c) there exists $A \in \mathfrak{g}_{\phi}$ such that $\mu_{0}=\lim _{t \rightarrow \infty} \exp (t A) . \mu$;

(d) such an $A$ can be chosen symmetric, with integer eigenvalues. 
Proof. 1. The group $G_{\phi}$ is a real reductive algebraic group with the Lie algebra $\mathfrak{g}_{\phi}$, which has a Cartan decomposition compatible with $\langle\cdot, \cdot\rangle$ (on the symmetric and the skew-symmetric endomorphisms). The equivalence of (ii) and (iv) follows directly from [RS, Theorem 4.4]. The equivalence of (ii) and (iii) follows from [RS, Theorem 4.3] and the fact that the group $G_{\phi}$ is the same for all the points of the orbit $G_{\phi} \cdot \mu$ (assertion 1 of Lemma 1). The implication (iii) $\Rightarrow(\mathrm{i})$ and the fact that the nilsoliton inner product has the required form follow from assertion 3 of Lemma 1

To prove the converse implication (i) $\Rightarrow($ iii), suppose that $\mathfrak{n}$ is an Einstein nilradical. Then for some $g \in \operatorname{GL}(n)$, the inner product $\langle\cdot, \cdot\rangle$ is nilsoliton on $\left(\mathbb{R}^{n}, g . \mu\right)$. We want to show that $g$ can be chosen from the group $G_{\phi}$. By (6) and assertion 2 of Theorem 1, for any nilsoliton inner product there exists a symmetric pre-Einstein derivation, so by conjugation by an element of $\operatorname{Aut}(\mathfrak{n})$ we can assume that there exists a nilsoliton inner product $\langle\cdot, \cdot\rangle^{\prime}$, with respect to which $\phi$ is symmetric. It follows that for all $X, Y \in \mathbb{R}^{n},\langle X, Y\rangle^{\prime}=\langle h X, Y\rangle$ for some positive definite $h$ symmetric with respect to $\langle\cdot, \cdot\rangle$ and belonging to $H$, the centralizer of $\exp \phi$ in $\mathrm{GL}^{+}(n)$. Then there exists $h_{1} \in H$, with all the eigenvalues positive, such that $h_{1}^{*} h_{1}=h\left(h_{1}^{*}\right.$ is the adjoint with respect to $\langle\cdot, \cdot\rangle)$. Hence $\langle\cdot, \cdot\rangle$ is a nilsoliton inner product on the Lie algebra $\left(\mathbb{R}^{n}, h_{1} . \mu\right)$. The two-dimensional abelian Lie group $H_{0}=\exp (\operatorname{Span}(\mathrm{id}, \phi))$ lies in the center of $H$, and $H=G_{\phi} H_{0}$, so $h_{1}=g g_{0}$ for some $g \in G_{\phi}$ and $g_{0} \in H_{0}$ (in fact, $g$ even belongs to $\exp \left(\mathfrak{g}_{\phi}\right)$ ). Moreover, $g_{0}=e^{t} \exp (s \phi)$ for some $t, s \in \mathbb{R}$. As $\exp (s \phi)$ is an automorphism of $\mu$ and $e^{t}$ is a scaling, the inner product $\langle\cdot, \cdot\rangle$ is nilsoliton on $\left(\mathbb{R}^{n}, g . \mu\right)$, with $g \in G_{\phi}$, which implies that $g$ is a critical point of $f$.

2. The fact that the closure of $G_{\phi} . \mu$ contains a unique closed orbit $G_{\phi} . \mu_{0}$ follows from [RS, 9.3]. By [RS, Lemma 3.3], there exists $A \in \mathfrak{g}_{\phi}$ such that $\mu_{0}=\lim _{t \rightarrow \infty} \exp (t A) . \mu$. Such an $A$ can be chosen symmetric with respect to $\langle\cdot, \cdot\rangle$ and also such that the subgroup $\exp (t A) \in G_{\phi}$ is algebraic, which implies that the eigenvalues of some nonzero multiple of $A$ are integers. This proves (c) and (d).

To prove (a) and (b), note that $\mu_{0}$, being a limit of nilpotent Lie brackets, is a nilpotent Lie bracket itself. Regardless of whether $\phi$ is or is not a pre-Einstein derivation for the nilpotent Lie algebra $\mathfrak{n}_{0}=\left(\mathbb{R}^{n}, \mu_{0}\right)$, it follows from RS, Theorems $4.3,4.4]$ that the function $\tilde{f}: G_{\phi} \rightarrow \mathbb{R}$ defined by $\tilde{f}(g)=\left\|g \cdot \mu_{0}\right\|^{2}$ has a critical point. Then by assertion 3(a) of Lemma 1, the algebra $\mathfrak{n}_{0}$ is an Einstein nilradical. It is not isomorphic to $\mathfrak{n}$, as otherwise $\mathfrak{n}$ would be an Einstein nilradical and its $G_{\phi}$-orbit would be closed by Theorem 5, 1(ii) above. This proves (a).

By assertion 3(b) of Lemma 1, either $\mathfrak{n}_{0}$ is abelian or $\phi=a \phi_{0}$, where $\phi_{0}$ is a pre-Einstein derivation for $\mathfrak{n}_{0}$. As $\operatorname{Tr} \phi^{2}=\operatorname{Tr} \phi$ and $\operatorname{Tr} \phi_{0}^{2}=\operatorname{Tr} \phi_{0}$ by (1), it follows that either $\phi=\phi_{0}, \operatorname{Tr} \phi_{0}=0$, or $\phi=0$. The equation $\operatorname{Tr} \phi_{0}=0$ is not possible, as $\mathfrak{n}_{0}$ is an Einstein nilradical, so $\phi_{0}>0$ (by 2 of Theorem 1). If $\phi=0$, then $G_{\phi}=\operatorname{SL}(n)$, so $\mathfrak{n}_{0}$ is abelian by [La3, Theorem 8.2] and the uniqueness of $\mu_{0}$.

Remark 2. One possible reason why in assertion 2(a) of Theorem 5 the limiting algebra $\mathfrak{n}_{0}$ can be abelian is that there is no Einstein nilradical whose pre-Einstein derivation is $\phi$. This could happen, for instance, when $\phi$ is nonpositive (assertion 2 of Theorem (1) or when $\phi>0$ but the condition of [Ni2, Lemma 1] is violated. The latter case is illustrated by the majority of free nilpotent Lie algebras. The pre-Einstein derivation $\phi$ of the $p$-step nilpotent free Lie algebra $\mathfrak{f}(m, p)$ on $m$ generators is a positive multiple of the derivation having eigenvalues $1,2, \ldots, p$ whose eigenspaces are the homogeneous components of $\mathfrak{f}(m, p)$. Although both inequalities (2) are satisfied, $\mathfrak{f}(m, p)$ is not an Einstein nilradical when say $m>2$ and $p>3$, 
as no Einstein nilradical can have such pre-Einstein derivation [Ni2, Lemma 2]. So for all $\mathfrak{n}=\mathfrak{f}(m, p)$, which are not Einstein nilradicals, the algebra $\mathfrak{n}_{0}$ is abelian.

We emphasize that the property of a nilpotent algebra $\mathfrak{n}=\left(\mathbb{R}^{n}, \mu\right)$ to be an Einstein nilradical depends on the closedness of the orbit of $\mu$ by the action of the group $G_{\phi}$ depending on $\mathfrak{n}$. As the result, the closure of a nonclosed orbit $G_{\phi} . \mu \subset \mathcal{V}$ may contain more than one Einstein nilradical. For each of them, the orbit of the Lie bracket under the action of its own group is closed, but the $G_{\phi^{-}}$orbit is closed only for one of them (assertion 2 of Theorem [5).

Example 2. Let $\mathfrak{n}^{\prime}=\left(\mathbb{R}^{m}, \mu^{\prime}\right)$ be a characteristically nilpotent Lie algebra. Its preEinstein derivation $\phi^{\prime}$ is zero, $G_{\phi^{\prime}}=\mathrm{SL}(m)$, and the closure of the orbit $G_{\phi^{\prime}} \cdot \mu^{\prime}$ contains the abelian algebra $\mathbb{R}^{m}$. Let a nilpotent algebra $\mathfrak{n}=\left(\mathbb{R}^{m+3}, \mu\right)$ be the direct sum of $\mathfrak{n}^{\prime}$ and the three-dimensional Heisenberg algebra $\mathfrak{h}_{3}$ given by $\left[X_{1}, X_{2}\right]=X_{3}$. The pre-Einstein derivation for $\mathfrak{h}_{3}$ is $\phi_{2}=\frac{2}{3} \operatorname{diag}(1,1,2)$. By assertion a of Theorem 7 below, the derivation $\phi=0_{\mathfrak{n}^{\prime}} \oplus \phi_{2}$ is a pre-Einstein derivation for $\mathfrak{n}$. As $\phi$ is not positive, the closure of $G_{\phi} \cdot \mu$ contains the abelian algebra $\mathbb{R}^{m+3}$. On the other hand, that closure also contains a nonabelian Einstein nilradical $\hat{\mathfrak{n}}=\left(\mathbb{R}^{m+3}, \hat{\mu}\right)$, the direct sum of $\mathbb{R}^{m}$ and $\mathfrak{h}_{3}$. The derivation $\hat{\phi}=\operatorname{id}_{\mathfrak{n}^{\prime}} \oplus \phi_{2}$ is pre-Einstein for $\hat{\mathfrak{n}}$, the $G_{\hat{\phi}^{-}}$orbit of $\hat{\mu}$ is closed, but the $G_{\phi^{-}}$orbit is not.

Remark 3. As follows from [RS, Theorem 4.3], if $g_{0} \in G_{\phi}$ is a critical point of the function $f$, then the set of minimal brackets (the brackets with the smallest norm) in the orbit $G_{\phi} \cdot \mu$ is $U_{\phi} \cdot g_{0} \cdot \mu$, where $U_{\phi}=G_{\phi} \cap \mathrm{SO}(n)$. This implies that the set of critical points of $f$ is $U_{\phi} g_{0}\left(\operatorname{Aut}(\mathfrak{n}) \cap G_{\phi}\right)$.

Theorems 1 and 2 suggest the following strategy for deciding whether a given nilpotent Lie algebra $\mathfrak{n}$ is an Einstein nilradical:

(a) Find a pre-Einstein derivation $\phi$ for $\mathfrak{n}$. In practice, this can be done just by solving a system of linear equations. As it follows from the proof of assertion 1(a) of Theorem 1, every maximal torus of $\operatorname{Der}(\mathfrak{n})$ contains a pre-Einstein derivation, so one can choose a particular maximal torus to reduce the amount of calculations. Also note that if $\phi$ is a (real, semisimple) candidate for being a pre-Einstein derivation, it suffices to check the validity of (11) only for semisimple derivations $\psi$ commuting with $\phi$. Indeed, $\operatorname{ad}_{\phi}$ is a real, semisimple endomorphism of $\operatorname{Der}(\mathfrak{n})$. If $\operatorname{ad}_{\phi} \psi=\lambda \psi$, with $\lambda \neq 0$, then both sides of (11) vanish, so it suffices to consider only those $\psi$ which commute with $\phi$. The nilpotent part $\psi^{N}$ of such a $\psi$ is a derivation commuting with $\phi$, so the eigenspaces of $\phi$ are invariant subspaces of $\psi^{N}$ and the restriction of $\psi^{N}$ to each of them is nilpotent, so for $\psi=\psi^{N}$, both sides of (1) again vanish.

(b) If the inequalities of (2) are not satisfied, then $\mathfrak{n}$ is not an Einstein nilradical. Otherwise, compute the algebra $\mathfrak{g}_{\phi}$ and use Theorem 2 or Theorem 5.

(c) Sometimes instead of (3) it is easier to prove that $\mathfrak{n}$ is an Einstein nilradical by explicitly producing a nilsoliton inner product. The pre-Einstein derivation gives a substantial amount of information for finding it. First of all, by assertion 2 of Theorem 1, if a nilsoliton inner product exists, it can be chosen in such a way that the eigenspaces of $\phi$ are orthogonal. Secondly, the pre-Einstein derivation completely determines the eigenvalue type. Also, as by (4) $\operatorname{Tr}\left(\operatorname{Ric}_{\mathfrak{n}} \phi\right)=0$, it follows from (6) and Theorem 1 that an inner 
product on $\mathfrak{n}$ is nilsoliton if and only if $\operatorname{Ric}_{\mathfrak{n}}=c(\mathrm{id}-\phi)$ for some $c<0$, where the expression for $\mathrm{Ric}_{\mathfrak{n}}$ is given by the right-hand side of (5).

\section{Applications of Theorems 1 and 2}

In this section, we use Theorem 1 and Theorem 2 to prove the following three facts: Theorem 3, which gives an easy-to-check condition for a nilpotent Lie algebra with a nice basis to be an Einstein nilradical, Theorem [6, which says that the property of being an Einstein nilradical is, in fact, a property of the complexification of a real nilpotent Lie algebra, and Theorem 7 , which says that the direct sum of nilpotent Lie algebras is an Einstein nilradical if and only if the summands are Einstein nilradicals.

Proof of Theorem 3. In brief, the proof proceeds as follows. We fix a nice basis and compute the pre-Einstein derivation and the group $G_{\phi}$. Then we show that the closedness of the orbit of the diagonal subgroup of $G_{\phi}$ is completely controlled by the convexity condition (i) and that if there is a critical point somewhere on $G_{\phi}$, then there is one on the diagonal.

Let $\mathcal{B}=\left(X_{1}, \ldots, X_{n}\right)$ be a nice basis for a nonabelian nilpotent Lie algebra $\mathfrak{n}=\left(\mathbb{R}^{n}, \mu\right)$. Let $\Lambda=\left\{(i, j, k): i<j, c_{i j}^{k} \neq 0\right\}$, \# $\Lambda=m>0$. In a Euclidean space $\mathbb{R}^{n}$ with the inner product $(\cdot, \cdot)$ and an orthonormal basis $f_{1}, \ldots, f_{n}$, define the finite subset $\mathbf{F}=\left\{Y_{a}=f_{i}+f_{j}-f_{k}: a=(i, j, k) \in \Lambda\right\}$ and denote by $L$ the affine span of $\mathbf{F}$, the smallest affine subspace of $\mathbb{R}^{n}$ containing $\mathbf{F}$. Fix an arbitrary ordering of the set $\Lambda$ and define an $m \times n$ matrix $Y$ whose $a$-th row has 1 in the columns $i$ and $j,-1$ in the column $k$, and zero elsewhere, where $a=(i, j, k) \in \Lambda$.

The proof follows the steps at the end of Section 3 .

Fix the nice basis $\mathcal{B}$ and an inner product $\langle\cdot, \cdot\rangle$ on $\mathfrak{n}$, with respect to which the nice basis is orthonormal. We say that an operator (an inner product) is diagonal if its matrix with respect to $\mathcal{B}$ is diagonal. For a vector $v=\left(v_{1}, \ldots, v_{n}\right)^{t} \in \mathbb{R}^{n}$, denote by $v^{D}$ the diagonal operator defined by $v^{D} X_{i}:=v_{i} X_{i}$. Note that $v^{D} \in \operatorname{Der}(\mathfrak{n})$ if and only if $v \in \operatorname{Ker} Y$. Similarly, for a vector $r=\left(r_{1}, \ldots, r_{n}\right)^{t} \in \mathbb{R}^{n}$ with $r_{i} \neq 0$, denote by $\langle\cdot, \cdot\rangle_{r}$ the diagonal inner product defined by $\left\langle X_{i}, X_{j}\right\rangle_{r}:=r_{i}^{2} \delta_{i j}$.

The Ricci operator $\operatorname{Ric}_{r}$ of the metric algebra $\left(\mathfrak{n},\langle\cdot, \cdot\rangle_{r}\right)$ is diagonal and

$$
\operatorname{Ric}_{r}=\left(-\frac{1}{2} Y^{t} \beta\right)^{D}
$$

for a vector $\beta \in \mathbb{R}^{m}$ with $\beta_{a}=\left(c_{i j}^{k} r_{i}^{-1} r_{j}^{-1} r_{k}\right)^{2}$, where $a=(i, j, k) \in \Lambda$. To see that, choose the $\langle\cdot, \cdot\rangle_{r}$-orthonormal basis $E_{i}=r_{i}^{-1} X_{i}$. Then by (5) and Definition 3, $\left\langle\operatorname{Ric}_{r} E_{k}, E_{l}\right\rangle_{r}=0$ when $k \neq l$, and the diagonal elements of $\mathrm{Ric}_{r}$ are $\left\langle\operatorname{Ric}_{r} E_{k}, E_{k}\right\rangle_{r}=\frac{1}{2} \sum_{i, j:(i, j, k) \in \Lambda}\left\langle\left[E_{i}, E_{j}\right], E_{k}\right\rangle_{r}^{2}-\frac{1}{2} \sum_{i, j:(k, i, j) \in \Lambda}\left\langle\left[E_{k}, E_{i}\right], E_{j}\right\rangle_{r}^{2}-$ $\frac{1}{2} \sum_{i, j:(i, k, j) \in \Lambda}\left\langle\left[E_{k}, E_{i}\right], E_{j}\right\rangle_{r}^{2}=-\frac{1}{2}\left(Y^{t} \beta\right)_{k}$, as $\left\langle\left[E_{i}, E_{j}\right], E_{k}\right\rangle_{r}=c_{i j}^{k} r_{i}^{-1} r_{j}^{-1} r_{k}$ and the $k$-th row of $Y^{t}$ has -1 in the columns $a=(i, j, k) \in \Lambda$ and 1 in the columns $a=(i, k, j) \in \Lambda$ and $a=(k, i, j) \in \Lambda$ (and 0 elsewhere).

We want to show that $\mathfrak{n}$ admits a diagonal pre-Einstein derivation (note that $\mathfrak{n}$ may have more than one nice basis). Let $\phi$ be a diagonal derivation satisfying (1) for all diagonal derivations $\psi$ (such a $\phi$ exists and is unique, as the left-hand side of (11) is a positive definite bilinear form on the space of diagonal derivations). Then $\phi=v^{D}$, with $v \in \operatorname{Ker} Y$, and $(v, u)=\left([1]_{n}, u\right)$, for all $u \in \operatorname{Ker} Y$. It follows that $v=[1]_{n}+Y^{t} \gamma$ for some $\gamma \in \mathbb{R}^{m}$ and that $0=Y v=[1]_{m}+Y Y^{t} \gamma$, so $\gamma=-\alpha$, where $\alpha$ is an arbitrary solution of the equation $Y Y^{t} \alpha=[1]_{m}$ from (ii) of the theorem (at 
this point we make no assumptions on the positivity of $\alpha$ ). The fact that such an $\alpha$ exists follows from the existence of $\phi$. As the symmetric matrix $Y Y^{t}$ is nonnegative, two solutions of the equation $Y Y^{t} \alpha=[1]_{m}$ may differ only by a vector from $\operatorname{Ker} Y^{t}$, so, although $\alpha$ may be not unique, the vector $v=[1]_{n}+Y^{t} \gamma=[1]_{n}-Y^{t} \alpha$ is welldefined. Hence

$$
\phi=\mathrm{id}-\left(Y^{t} \alpha\right)^{D} .
$$

To show that such a $\phi$ is indeed a pre-Einstein derivation, consider an arbitrary $\psi \in \operatorname{Der}(\mathfrak{n})$. By (4) $\operatorname{Tr}(\operatorname{Ric} \psi)=0$, for any inner product on $\mathfrak{n}$. In particular, for any diagonal inner product $\langle\cdot, \cdot\rangle_{r}$, equation (10) implies that $\operatorname{Tr}\left(\left(Y^{t} \beta\right)^{D} \psi\right)=0$. Choose $v \in \mathbb{R}^{n}$ in such a way that $v^{D}$ and $\psi$ have the same diagonal entries. Then $0=\operatorname{Tr}\left(\left(Y^{t} \beta\right)^{D} v^{D}\right)=(\beta, Y v)$. This holds for any vector $\beta \in \mathbb{R}^{m}$ with the components $\beta_{a}=\left(c_{i j}^{k} r_{i}^{-1} r_{j}^{-1} r_{k}\right)^{2}$, where $a=(i, j, k) \in \Lambda$. Choose one such $a=(i, j, k)$ and take $r_{i}=r_{j}=e^{-t}$, and $r_{l}=1$ for $l \neq i, j$. Substituting this to $(\beta, Y v)=0$, dividing by $e^{4 t}$ and taking the limit when $t \rightarrow \infty$, we obtain $(Y v)_{a}=0$. It follows that $Y v=0$, so that $v^{D}$, the projection of $\psi$ to the diagonal, is also a derivation. Then $\operatorname{Tr}(\phi \psi)=\operatorname{Tr}\left(\phi v^{D}\right)=\operatorname{Tr} v^{D}=\operatorname{Tr} \psi$, so the derivation $\phi$ given by (11) (which is clearly semisimple and real) is indeed a pre-Einstein derivation.

As in Theorem 2, let $\mathfrak{g}_{\phi}$ be the Lie algebra defined by (3), $G_{\phi}$ be the Lie group defined by (9), and $f: G_{\phi} \rightarrow \mathbb{R}$ be the function defined by $f(g)=\|g \cdot \mu\|^{2}$, where $\mu$ is the Lie bracket of $\mathfrak{n}$.

We first consider the behavior of $f$ restricted to the diagonal subgroup of $G_{\phi}$. Let $\mathfrak{g}_{D}$ be the abelian subalgebra consisting of all the diagonal elements of $\mathfrak{g}_{\phi}$.

Lemma 2. 1. The following three conditions are equivalent:

(i) The projection of the origin of $\mathbb{R}^{n}$ to $L$ lies in the interior of $\operatorname{Conv}(\mathbf{F})$.

(ii) There exists a vector $\alpha \in \mathbb{R}^{m}$ with positive coordinates satisfying $Y Y^{t} \alpha=$ $[1]_{m}$.

(iii) The function $f_{D}: \mathfrak{g}_{D} \rightarrow \mathbb{R}$ defined by $f_{D}(A)=f(\exp (A))$ for $A \in \mathfrak{g}_{D}$ has a critical point.

2. If $A \in \mathfrak{g}_{D}$ is a critical point of $f_{D}: \mathfrak{g}_{D} \rightarrow \mathbb{R}$, then $\exp (A)$ is a critical point of $f: G_{\phi} \rightarrow \mathbb{R}$, so $\mathfrak{n}$ is an Einstein nilradical by Theorem 2 ,

Proof. 1. The equivalence of the geometric condition (i) and the algebraic condition (ii) is an easy linear algebraic argument (see the proof of Corollary 1 in [Ni2]).

To show that (i) and (ii) are equivalent to (iii), we start with the following observation: let $\left\{w_{a}\right\}$ be a finite set of vectors in a Euclidean space $V$ with $\operatorname{Span}\left(w_{a}\right)=V^{\prime} \subset V$, and let $b_{a}$ be positive numbers. Then the function $e: V \rightarrow \mathbb{R}$ defined by $e(x)=\sum_{a} b_{a} e^{\left(w_{a}, x\right)}$ has a critical point if and only if it attains its minimum if and only if the origin of $V$ lies in the interior (relative to $V^{\prime}$ ) of the convex hull of the vectors $w_{a}$. Since $e(x+y)=e(x)$ for $y \perp V^{\prime}$, we lose no generality by replacing $V$ by $V^{\prime}$ and $e$ by $e_{\mid V^{\prime}}$. The fact that a critical point, if it exists, is a minimum, is clear, as $e(x)$ is positive and convex. Next, at a critical point $x, \operatorname{de}(x)=\sum_{a} b_{a} e^{\left(w_{a}, x\right)} w_{a}=0$, so 0 lies in the interior of the convex hull of the $w_{a}$ 's. Conversely, if $M$ is a large positive number, the preimage $e^{-1}(0, M]$ is closed, nonempty, and is contained in the convex polyhedron $\bigcap_{a}\left\{x:\left(w_{a}, x\right) \leq \ln \left(M b_{a}^{-1}\right)\right\}$. If the origin lies in the interior of the convex hull of the $w_{a}$ 's, that polyhedron is bounded, so $e^{-1}(0, M]$ is compact, which proves the claim. 
By (11), $\mathfrak{g}_{D}=\left\{v^{D}:\left(v,[1]_{n}\right)=\left(v, Y^{t} \alpha\right)=0\right\}$. Then $f_{D}\left(v^{D}\right)=\left\|\exp \left(v^{D}\right) \cdot \mu\right\|^{2}=$ $\sum_{a=(i, j, k) \in \Lambda}\left(c_{i j}^{k}\right)^{2} \exp \left(2\left(v_{k}-v_{i}-v_{j}\right)\right)=\sum_{a=(i, j, k) \in \Lambda}\left(c_{i j}^{k}\right)^{2} \exp \left(-2(Y v)_{a}\right)$. By the observation from the previous paragraph, with $V=\left\{v:\left(v,[1]_{n}\right)=\left(v, Y^{t} \alpha\right)=0\right\}$ and $w_{a}=Y_{a}-P$, the projections of the vectors $Y_{a}$ to $V$, we find that $f_{D}$ has a critical point if and only if the origin lies in the interior of the convex hull of the vectors $Y_{a}-P$; that is, if and only if $P$ lies in the interior of $\operatorname{Conv}(\mathbf{F})$.

2. Let $v^{D} \in \mathfrak{g}_{D}$ be a critical point of $f_{D}$ and let $\mu^{\prime}=\exp \left(v^{D}\right) . \mu$. The basis $\mathcal{B}$ is still nice for the Lie algebra $\mathfrak{n}^{\prime}=\left(\mathbb{R}^{n}, \mu^{\prime}\right)$, so by (10) the Ricci operator $\mathrm{Ric}_{\mathfrak{n}^{\prime}}$ of the metric Lie algebra $\left(\mathfrak{n}^{\prime},\langle\cdot, \cdot\rangle\right)$ is diagonal. By 2(a) of Lemma 1 $\left.\frac{d}{d t}\right|_{t=0} f\left(\exp (t A) \cdot \exp \left(v^{D}\right)\right)=8 \operatorname{Tr}\left(\operatorname{Ric}_{\mathfrak{n}^{\prime}} A\right)$, for any $A \in \operatorname{End}(\mathfrak{n})$. The expression on the right-hand side vanishes for all the $A$ 's with the zero diagonal, as $\mathrm{Ric}_{\mathfrak{n}^{\prime}}$ is diagonal, and for all $A \in \mathfrak{g}_{D}$, as $v^{D}$ is critical for $f_{D}$. Hence $\exp \left(v^{D}\right)$ is a critical point of $f$.

Lemma 2 proves the "if" part of the theorem. To prove the "only if" part, we will show that if the function $f$ has a critical point somewhere on $G_{\phi}$, then it has a critical point on $\exp \mathfrak{g}_{D}$.

We will need the following lemma, which slightly refines the Cartan decomposition of the reductive real algebraic group $G_{\phi}$. On the Lie algebra level, we have $\mathfrak{g}_{\phi}=\mathfrak{u}_{\phi} \oplus \mathfrak{p}=\mathfrak{u}_{\phi} \oplus \mathfrak{p}_{0} \oplus \mathfrak{g}_{D}$, where $\mathfrak{u}_{\phi}$ is the subalgebra of all the skew-symmetric matrices from $\mathfrak{g}_{\phi}, \mathfrak{p}$ is the subspace of all the symmetric matrices from $\mathfrak{g}_{\phi}$, and $\mathfrak{p}_{0}$ is the subspace of all the matrices with the zero diagonal from $\mathfrak{p}$. On the Lie group level, let $U_{\phi}=\mathrm{O}(n) \cap G_{\phi}$, a maximal compact subgroup of $G_{\phi}$, let $G_{D}=\exp \mathfrak{g}_{D}$, a maximal torus in $G_{\phi}$, and let $P_{0}=\exp \mathfrak{p}_{0}$.

Lemma 3. The map $U_{\phi} \times P_{0} \times G_{D} \rightarrow G_{\phi}$ defined by $\left(u, p, g_{D}\right) \rightarrow u_{p} g_{D}$ for $u \in U_{\phi}, p \in P_{0}$ and $g_{D} \in G_{D}$ is surjective.

Proof of Lemma 3. We follow the approach of [WZ. Let $\pi(g)=g^{t} g$ for $g \in G_{\phi}$, and let $M=\pi\left(G_{\phi}\right)$. Clearly, $\pi\left(g_{1}\right)=\pi\left(g_{2}\right)$ if and only if $g_{2}=u g_{1}$ for some $u \in U_{\phi}$. The action of $G_{\phi}$ on itself from the right defines the action on the homogeneous space $M=G_{\phi} / U_{\phi}$ by $x \rightarrow g^{t} x g$ for $x \in M$. An inner product $Q$ on $\mathfrak{g}_{\phi}$ defined by $Q\left(A_{1}, A_{2}\right)=\operatorname{Tr}\left(A_{1} A_{2}^{t}\right)$ is $\operatorname{Ad}\left(U_{\phi}\right)$-invariant and gives, by right translations, a bi-invariant Riemannian metric on $M$. The space $M$ with that metric is a Hadamard symmetric space with the de Rham decomposition $\prod_{j=1}^{p} \mathrm{SL}\left(d_{j}\right) / \mathrm{SO}\left(d_{j}\right) \times \mathbb{R}^{p-2}$, where $d_{1}, \ldots, d_{p}$ are the multiplicities of the eigenvalues of the pre-Einstein derivation $\phi$. The space $\mathfrak{g}_{D}$ is a maximal abelian subalgebra in $\mathfrak{p}=\mathfrak{u}_{\phi}^{\perp}$, so $M_{D}=\pi\left(\exp \mathfrak{g}_{D}\right) \subset M$ is a complete flat totally geodesic submanifold. For any $g \in G$, let $\gamma=\gamma(s)$ be (the unique) geodesic realizing the distance $d(g)$ from $\pi(g)$ to $M_{D}$, with $s \in[0, d(g)]$ an arclength parameter, such that $\gamma(0)=\pi\left(g_{D}\right) \in M_{D}, \gamma(d(g))=\pi(g)$. Then $g_{D}^{-1} \gamma(s) g_{D}^{-1}$ is a geodesic of $M$ passing through the basepoint $o=\pi(\mathrm{id}) \in M$ whose tangent vector is orthogonal to the tangent space of $M_{D}$ at $o$. It follows that $g_{D}^{-1} \gamma(s) g_{D}^{-1}=\pi(\exp (s A))$ for some symmetric matrix $A \in \mathfrak{g}_{\phi}$ such that $Q\left(A, \mathfrak{g}_{D}\right)=0$, that is, for some $A \in \mathfrak{p}_{0}$. So $\pi(g)=g_{D} \exp (d(g) A) g_{D}=\pi\left(\exp \left(\frac{1}{2} d(g) A\right) g_{D}\right)$, as required.

Assume that $\mathfrak{n}$ is an Einstein nilradical and that $g_{0} \in G_{\phi}$ is a critical point of the function $f$. By Lemma $3 g_{0}=u p g_{D}$ for some $u \in U_{\phi}, p \in P_{0}$ and $g_{D} \in G_{D}$. As $f(u g)=f(g)$ for any $u \in U_{\phi}$ and $g \in G_{\phi}$, the point $g_{1}=p g_{D}$ is also critical. 
Denote $\mu^{\prime}=\exp \left(g_{D}\right) \cdot \mu$ and $\mathfrak{n}^{\prime}=\left(\mathbb{R}^{n}, \mu^{\prime}\right)$. The basis $\mathcal{B}$ is still a nice basis for $\mathfrak{n}^{\prime}$. Moreover, by 1 of Lemma 1, $\phi$ is a pre-Einstein derivation of $\mathfrak{n}^{\prime}$, the algebra $\mathfrak{g}_{\phi}$ and the group $G_{\phi}$ for $\mathfrak{n}^{\prime}$ are the same as those for $\mathfrak{n}$, and $f_{\mathfrak{n}^{\prime}}(g)=f_{\mathfrak{n}}\left(g g_{D}\right)$ for $g \in G_{\phi}$. We can therefore replace $\mathfrak{n}$ by $\mathfrak{n}^{\prime}$ and assume that the function $f$ has a critical point $p \in P_{0}$. Then $p=\exp A$ for some $A \in \mathfrak{p}_{0}$. Consider a function $F(t)=f(\exp (t A))$ for $t \in \mathbb{R}$. The function $F$ has a critical point at $t=1$, as $\exp (A)$ is critical for $f$, and at $t=0$, as by 2(a) of Lemma 1, $F^{\prime}(0)=\left.\frac{d}{d t}\right|_{t=0} f(\exp (t A))=$ $8 \operatorname{Tr}\left(\operatorname{Ric}_{\mathfrak{n}} A\right)=0$, since $\operatorname{Ric}_{\mathfrak{n}}$ is diagonal by (10). Let $\left\{E_{i}\right\}$ be an orthonormal basis of the eigenvectors of the symmetric matrix $A$, with the corresponding eigenvalues $\nu_{i}$, and let $C_{i j}^{k}=\left\langle\left[E_{i}, E_{j}\right], E_{k}\right\rangle$ be the structural constants with respect to the basis $\left\{E_{i}\right\}$. Then $F(t)=f(\exp (t A))=\sum_{i, j, k}\left(C_{i j}^{k}\right)^{2} \exp \left(2 t\left(\nu_{i}+\nu_{j}-\nu_{k}\right)\right)$, so $F^{\prime \prime}(t) \geq 0$. Since the convex function $F(t)$ has two critical points, it must be a constant, which implies that $\nu_{i}+\nu_{j}=\nu_{k}$ for all the triples $(i, j, k)$ with $C_{i j}^{k} \neq 0$, so $A \in \operatorname{Der}(\mathfrak{n})$ and $p \in \operatorname{Aut}(\mathfrak{n})$. As $f(g)=f\left(g p^{-1}\right)$ for any $p \in \operatorname{Aut}(\mathfrak{n})$ and $g \in G_{\phi}$, the identity is also a critical point for $f$.

Thus if $\mathfrak{n}$ is an Einstein nilradical, then the function $f$ has a critical point on $\exp \mathfrak{g}_{D}$, which proves the "only if" part of the theorem by assertion 1 of Lemma 2 ,

The above proof shows that if a nilpotent Lie algebra with a nice basis is an Einstein nilradical, then the nilsoliton inner product can be chosen to be diagonal. This is by no means obvious, as a nilpotent Lie algebra can have two quite different nice bases, the easiest example being a direct sum of two copies of the Heisenberg algebra $\mathfrak{h}_{3}$ given by $\left[X_{1}, X_{2}\right]=X_{5},\left[X_{3}, X_{4}\right]=X_{6}$. The basis $\left\{X_{1} \pm X_{3}, X_{2} \pm\right.$ $\left.X_{4}, X_{5} \pm X_{6}\right\}$ for this algebra is also nice, with a different number of nonzero structural constants.

A nilsoliton inner product for a nilpotent Lie algebra with a nice basis can sometimes be found explicitly. For instance, from (10, 11), if rk $Y=m$, the inner product $\langle\cdot, \cdot\rangle_{r}$ is nilsoliton if we choose $r=\left(e^{s_{1}}, \ldots, e^{s_{n}}\right) \in \mathbb{R}^{n}$ in such a way that the $s_{i}$ 's satisfy the linear system $(Y s)_{a}=\sqrt{\alpha_{a}}\left|c_{i j}^{k}\right|^{-1}$ for $a=(i, j, k) \in \Lambda$.

There exist many nilpotent Lie algebras having no nice basis, as the following two examples show.

Example 3. Let $1 \leq p \leq \frac{1}{2} q(q-1)$ and let $X_{1}, \ldots, X_{q}$ and $X_{q+1}, \ldots, X_{q+p}$ be bases for $\mathbb{R}^{q}$ and for $\mathbb{R}^{p}$, respectively. Consider all the two-step nilpotent Lie algebras $\mathfrak{n}_{\mathcal{B}}=\left(\mathbb{R}^{q+p}, \mu\right)$ of type $(p, q)$ (with $\left.[\mathfrak{n}, \mathfrak{n}]=\mathbb{R}^{p}\right)$, for which the basis $X_{1}, \ldots, X_{q+p}$ is nice. Every such algebra is defined by a choice of the set $\Lambda=\{(i, j, k): 1 \leq$ $\left.i<j \leq q \leq k, c_{i j}^{k} \neq 0\right\}$ and then by a choice of $\# \Lambda$ nonzero numbers $c_{i j}^{k}$. There exists a finite number of the possible choices for $\Lambda$. Moreover, from Definition 3 it follows that for any such $\Lambda$, \# $\Lambda \leq m(p, q)=\min \left(\frac{1}{2} q(q-1), \frac{1}{2} p q\right)$. So every such algebra $\mathfrak{n}_{\mathcal{B}}$ is defined by a point in a finite union $\mathcal{S}$ of linear subspaces of the space $\mathcal{V}(p, q)=\left(\wedge^{2} \mathbb{R}^{q}\right)^{p}$ (see Section 5), each of dimension at most $m(p, q)$. The set of points of $\mathcal{V}(p, q)$ defining the two-step nilpotent algebras of type $(p, q)$ having a nice basis is the $\operatorname{GL}(q) \times \mathrm{GL}(p)$-orbit of $\mathcal{S}$ (if a nice basis exists for such an algebra, then there exists a nice basis which is a subset of $\left.\mathbb{R}^{q} \cup \mathbb{R}^{p}\right)$. From the dimension count (as in [Eb1]), the orbit of $\mathcal{S}$ cannot cover the whole space $\mathcal{V}(p, q)$ (and its subspace $\left.\mathcal{V}^{0}(p, q)\right)$, provided $m(p, q)+q^{2}+p^{2}-1<\frac{1}{2} p q(q-1)$. So for all the pairs $(p, q)$ satisfying the latter inequality, there exists a two-step nilpotent algebra of 
type $(p, q)$ having no nice basis. There is an infinite set of such pairs (for instance, the inequality holds for all $q-1 \geq p \geq 6$ ).

Example 4. Consider the free three-step nilpotent Lie algebra $\mathfrak{n}=\mathfrak{f}(3,3)$ on three generators (see Ni2 for details). Suppose $\mathcal{B}=\left\{X_{i}\right\}$ is a nice basis for $\mathfrak{n}$. As it follows from the definition, every bracket (and every multiple bracket) of the vectors from $\mathcal{B}$ is proportional to an element of $\mathcal{B}$ (possibly zero). In particular, $\mathcal{B} \cap[\mathfrak{n}, \mathfrak{n}]$ is a basis for $[\mathfrak{n}, \mathfrak{n}]$. Then the set $\mathcal{B} \backslash(\mathcal{B} \cap[\mathfrak{n}, \mathfrak{n}])$ (of cardinality $3=\operatorname{dim} \mathfrak{n}-\operatorname{dim}[\mathfrak{n}, \mathfrak{n}])$ spans a linear complement to $[\mathfrak{n}, \mathfrak{n}]$ in $\mathfrak{n}$, so its elements $X_{1}, X_{2}, X_{3}$ (up to relabeling) can be taken as the generators for $\mathfrak{n}$. From the Jacobi identity and the fact that every bracket $\left[\left[X_{i}, X_{j}\right], X_{k}\right],\{i, j, k\}=\{1,2,3\}$, is proportional to an element of the basis $\mathcal{B}$, it follows that $\operatorname{rk}\left(\left[\left[X_{1}, X_{2}\right], X_{3}\right],\left[\left[X_{3}, X_{1}\right], X_{2}\right],\left[\left[X_{2}, X_{3}\right], X_{1}\right]\right) \leq 1$. This contradicts the fact that the nine brackets $\left[\left[X_{i}, X_{j}\right], X_{k}\right], i, j, k \in\{1,2,3\}, i<j$, span the eight-dimensional space $[[\mathfrak{n}, \mathfrak{n}], \mathfrak{n}]$.

Another application of Theorems 1 and 2 is the following theorem.

Theorem 6. Let $\mathfrak{n}_{1}$ and $\mathfrak{n}_{2}$ be two (real) nilpotent Lie algebras whose complexifications are isomorphic as the complex nilpotent Lie algebras. If $\mathfrak{n}_{1}$ is an Einstein nilradical, then so is $\mathfrak{n}_{2}$, with the same eigenvalue type.

Note that two real nilpotent algebras with isomorphic complexifications might be quite different. For instance, two-step nilpotent algebras $\mathfrak{n}_{1}$ and $\mathfrak{n}_{2}$ defined by $\left[X_{1}, X_{2}\right]=Z_{1},\left[X_{3}, X_{4}\right]=Z_{2}$, and by $\left[X_{1}, X_{3}\right]=\left[X_{2}, X_{4}\right]=Z_{1},\left[X_{1}, X_{4}\right]=$ $\left[X_{3}, X_{2}\right]=Z_{2}$, respectively, are isomorphic over $\mathbb{C}$. However, the algebra $\mathfrak{n}_{1}$ is decomposable: it is a direct sum of two copies of the Heisenberg algebra $\mathfrak{h}_{3}$, while $\mathfrak{n}_{2}$ is nonsingular (for any $X \in \mathfrak{n}_{2} \backslash \mathfrak{z}$, where $\mathfrak{z}$ is the center of $\mathfrak{n}_{2}$, the $\operatorname{map}_{X}: \mathfrak{n}_{2} \rightarrow \mathfrak{z}$ is surjective [Eb2]).

Theorem [ 6 can be useful when one knows the classification of a family of nilpotent algebras only up to complex isomorphism (see e.g. GT or several lists of sevendimensional nilpotent Lie algebras available in the literature). Also, in the majority of the results of the Geometric Invariant Theory (which seems to be strongly present in the study of Einstein nilradicals), the ground field is $\mathbb{C}[\mathrm{VP}]$.

Proof of Theorem 6. We start with constructing a pre-Einstein derivation in the complex case, which we define as a semisimple derivation $\phi$ satisfying (11), and showing that a pre-Einstein derivation always exists, is unique up to conjugation by $\operatorname{Aut}(\mathfrak{n})$, and has all its eigenvalues rational. The proof is almost identical to that of assertion 1 of Theorem 1 (with the only difference being that we do not need to split the torus $\mathfrak{t}$ into the compact and the fully $\mathbb{R}$-reducible parts), so we omit it.

Now let $\mathfrak{n}=\left(\mathbb{R}^{n}, \mu\right)$ be a real nilpotent Lie algebra with the complexification $\mathfrak{n}^{\mathbb{C}}=\left(\mathbb{C}^{n}, \mu\right)$. Then $\operatorname{Der}\left(\mathfrak{n}^{\mathbb{C}}\right)=(\operatorname{Der}(\mathfrak{n}))^{\mathbb{C}}$, so the pre-Einstein derivation $\phi=\phi_{\mathfrak{n}}$ also serves as a pre-Einstein derivation for $\mathfrak{n}^{\mathbb{C}}$. It follows that the pre-Einstein derivations of two real Lie algebras whose complexifications are isomorphic have the same eigenvalues.

By Theorem 2 $\mathfrak{n}$ is an Einstein nilradical if and only if the orbit $G_{\phi} \cdot \mu$ is closed in $\mathcal{V}$. Let $\mathfrak{g}_{\phi}^{\mathbb{C}}$ be the complexification of the Lie algebra $\mathfrak{g}_{\phi}$ defined by (3), and let $G_{\phi}^{\mathbb{C}} \subset \operatorname{SL}(n, \mathbb{C})$ be the Lie group with the Lie algebra $\mathfrak{g}_{\phi}^{\mathbb{C}}$ (the group $G_{\phi}^{\mathbb{C}}$ is defined by the right-hand side of $(\underline{8})$, but over $\mathbb{C}$ ). Consider the orbit of $\mu$ in the space $\mathcal{V}^{\mathbb{C}}=\bigwedge^{2}\left(\mathbb{C}^{n}\right)^{*} \otimes \mathbb{C}^{n}$, the complexification of $\mathcal{V}$, under the action of $G_{\phi}^{\mathbb{C}}$. By 
the results of [BHC, Proposition 2.3] and [Bir, Corollary 5.3], the orbit $G_{\phi} \cdot \mu$ is (Euclidean) closed in $\mathcal{V}$ if and only if $G_{\phi}^{\mathbb{C}} \cdot \mu$ is Zariski-closed in $\mathcal{V}^{\mathbb{C}}$.

It follows that two real algebras having isomorphic complexifications either are or are not Einstein nilradicals simultaneously. In the former case, the eigenvalue types are the same, as the spectra of the pre-Einstein derivations are the same.

Remark 4. The proof shows that the property of a real nilpotent Lie algebra $\mathfrak{n}$ to be an Einstein nilradical is, in fact, a property of its complexification $\mathfrak{n}^{\mathbb{C}}$. Namely, call a complex nilpotent Lie algebra $\mathfrak{N}=\left(\mathbb{C}^{n}, \nu\right)$ with a pre-Einstein derivation $\phi$ stable, if the orbit $G_{\phi}^{\mathbb{C}} . \nu$ is Zariski-closed in $\mathcal{V}^{\mathbb{C}}$. Then $\mathfrak{n}$ is an Einstein nilradical if and only if $\mathfrak{n}^{\mathbb{C}}$ is stable.

Yet another application of Theorem 1 and Theorem 2 is the following theorem.

Theorem 7. Let a nilpotent Lie algebra $\mathfrak{n}$ be the direct sum of nilpotent Lie algebras $\mathfrak{n}_{1}$ and $\mathfrak{n}_{2}$. Then:

(a) If $\phi_{i}$ are pre-Einstein derivations for $\mathfrak{n}_{i}$, then $\phi=\phi_{1} \oplus \phi_{2}$ is a pre-Einstein derivation for $\mathfrak{n}$.

(b) The algebra $\mathfrak{n}$ is an Einstein nilradical if and only if both algebras $\mathfrak{n}_{1}$ and $\mathfrak{n}_{2}$ are Einstein nilradical.

As it follows from Pay, Theorem 4], if both algebras $\mathfrak{n}_{1}$ and $\mathfrak{n}_{2}$ are Einstein nilradicals, a nilsoliton inner product on $\mathfrak{n}$ can be taken as the orthogonal sum of (appropriately scaled) nilsoliton inner products on the $\mathfrak{n}_{i}$ 's. Geometrically this says that the Riemannian product of two Einstein solvmanifolds of the same Ricci curvature is again an Einstein solvmanifold.

In the case when one of the summands is abelian, assertion (b) follows from La2, Proposition 3.3]. Note also that assertion (b) is very far from being true for the semidirect sum: even when both summands are abelian, the resulting algebra $\mathfrak{n}$ is two-step nilpotent and can easily not be an Einstein nilradical (see Section 5).

Proof. In the proof, we use $\oplus$ in several different meanings: as a direct sum of linear spaces (even when the spaces are Lie algebras), as the direct sum of operators, and as the direct sum of Lie brackets (in the obvious sense). We use $\dot{+}$ for the direct sum of Lie algebras.

(a) It is not difficult to see that the algebra $\operatorname{Der}(\mathfrak{n})$ admits the following splitting into four subspaces: $\operatorname{Der}(\mathfrak{n})=\bigoplus_{i, j=1}^{2} \mathfrak{d}_{i j}$, where $\mathfrak{d}_{i i}, i=1,2$, is the space of all $\psi \in \operatorname{End}(\mathfrak{n})$ such that $\psi\left(\mathfrak{n}_{i}\right) \subset \mathfrak{n}_{i}, \psi_{\mid \mathfrak{n}_{i}} \in \operatorname{Der}\left(\mathfrak{n}_{i}\right), \psi_{\mid \mathfrak{n}_{j}}=0$, for $j \neq i$, and where $\mathfrak{d}_{i j}, i \neq j$, is the space of all $\psi \in \operatorname{End}(\mathfrak{n})$ such that $\psi_{\mid \mathfrak{n}_{j} \oplus\left[\mathfrak{n}_{i}, \mathfrak{n}_{i}\right]}=0$ and $\psi\left(\mathfrak{n}_{i}\right)$ lies in the center of $\mathfrak{n}_{j}$.

Now let $\phi \in \mathfrak{d}_{11} \oplus \mathfrak{d}_{22}$ be the derivation of $\mathfrak{n}$ such that $\phi_{\mid \mathfrak{n}_{i}}=\phi_{i}, i=1,2$. Clearly, $\phi$ is semisimple and real. Moreover, equation (1) holds for any $\psi \in \mathfrak{d}_{11} \oplus \mathfrak{d}_{22}$, as each of the $\phi_{i}$ 's is pre-Einstein, and for any $\psi \in \mathfrak{d}_{12} \oplus \mathfrak{d}_{21}$, as both sides vanish.

(b) The "if" part follows directly from [Pay, Theorem 4]. To prove the "only if" part, choose and fix the pre-Einstein derivations $\phi_{1}, \phi_{2}$, and $\phi=\phi_{1} \oplus \phi_{2}$ for $\mathfrak{n}_{1}, \mathfrak{n}_{2}$, and $\mathfrak{n}$, respectively, and consider the algebras $\mathfrak{g}_{\phi_{i}} \subset \mathfrak{s l}\left(\mathfrak{n}_{i}\right), i=1,2$, and $\mathfrak{g}_{\phi} \subset \mathfrak{s l}(\mathfrak{n})$, as in (3) . Note that $\mathfrak{g}_{\phi} \supset \mathfrak{g}_{\phi_{1}} \oplus \mathfrak{g}_{\phi_{2}}$. Let $\mathfrak{n}_{i}=\left(\mathbb{R}^{n_{i}}, \mu_{i}\right), i=1,2$; then $\mathfrak{n}=\left(\mathbb{R}^{n_{1}+n_{2}}, \mu\right)$, with $\mu=\mu_{1} \oplus \mu_{2}$.

Suppose that $\mathfrak{n}$ is an Einstein nilradical but $\mathfrak{n}_{1}$ is not. By assertion 2 of Theorem [5, there exists $A_{1} \in \mathfrak{g}_{\phi_{1}} \subset \mathfrak{s l}\left(\mathfrak{n}_{1}\right)$ such that the limit $\mu_{1}^{\prime}=\lim _{t \rightarrow \infty}\left(\exp \left(t A_{1}\right) \cdot \mu_{1}\right)$ 
exists, and the algebra $\mathfrak{n}_{1}^{\prime}=\left(\mathbb{R}^{n_{1}}, \mu_{1}^{\prime}\right)$ is not isomorphic to $\mathfrak{n}_{1}$. Then for $A=$ $A_{1} \oplus 0_{\mid \mathfrak{n}_{2}} \in \mathfrak{g}_{\phi}$, we have $\lim _{t \rightarrow \infty}(\exp (t A) . \mu)=\mu_{1}^{\prime} \oplus \mu_{2}$. As $\mathfrak{n}$ is an Einstein nilradical, the algebra $\mathfrak{n}^{\prime}=\mathfrak{n}_{1}^{\prime} \dot{+} \mathfrak{n}_{2}$ must be isomorphic to the algebra $\mathfrak{n}=\mathfrak{n}_{1} \dot{+} \mathfrak{n}_{2}$ by (ii) of Theorem 2 .

The claim now follows from the purely Lie-algebraic fact that if the algebras $\mathfrak{n}_{1}$ and $\mathfrak{n}_{1}^{\prime}$ are not isomorphic, then so are $\mathfrak{n}_{1} \dot{+} \mathfrak{n}_{2}$ and $\mathfrak{n}_{1}^{\prime} \dot{\dot{ }} \mathfrak{n}_{2}$, for any $\mathfrak{n}_{2}$, which in turn follows from the uniqueness of a decomposition of a Lie algebra into the direct sum of undecomposable ones, up to permutation and isomorphism. To decompose an arbitrary (in particular, a nilpotent) Lie algebra $\mathfrak{n}$, one can use the following approach. First, split off a direct abelian summand $\mathfrak{a}$, if it exists: $\mathfrak{n}=\widehat{\mathfrak{n}} \dot{+} \mathfrak{a}$, where $\mathfrak{a}$ is a linear complement to $[\mathfrak{n}, \mathfrak{n}]$ in the center $\mathfrak{z}(\mathfrak{n})$ and $\widehat{\mathfrak{n}} \supset[\mathfrak{n}, \mathfrak{n}]$ is a linear complement to $\mathfrak{a}$ in $\mathfrak{n}$. This decomposition is not in general unique, but it is unique up to a central automorphism of $\mathfrak{n}$, namely up to $h \in \operatorname{Aut}(\mathfrak{n})$ such that $(h-\mathrm{id})(\mathfrak{n}) \subset$ $\mathfrak{z}(\mathfrak{n})$ [RWZ, Theorem 2.4, Eq. (2.28)]. Next, when a particular $\widehat{\mathfrak{n}}$ is chosen (note that $\mathfrak{z}(\widehat{\mathfrak{n}}) \subset[\widehat{\mathfrak{n}}, \widehat{\mathfrak{n}}])$, the further decomposition is unique up to permutation by [RWZ, Theorem 2.8(e)]. It follows that a decomposition of a Lie algebra into the direct sum of undecomposable Lie algebras is unique, up to isomorphism and permutation, so the algebras $\mathfrak{n}_{1} \dot{+} \mathfrak{n}_{2}$ and $\mathfrak{n}_{1}^{\prime} \dot{+} \mathfrak{n}_{2}$, with $\mathfrak{n}_{1} \neq \mathfrak{n}_{1}^{\prime}$, are nonisomorphic.

\section{TWO-STEP EINSTEIN NILRADiCAlS}

In this section, the technique developed in the preceding sections is applied to the two-step nilpotent Lie algebras. We prove Theorem 4 and also consider some exceptional cases.

We start with some preliminary facts, mostly following [Eb2]. A two-step nilpotent Lie algebra $\mathfrak{n}$ of dimension $p+q$ is said to be of type $(p, q)$ if its derived algebra $\mathfrak{m}=[\mathfrak{n}, \mathfrak{n}]$ has dimension $p$. Clearly, $\mathfrak{m} \subset \mathfrak{z}(\mathfrak{n})$, the center of $\mathfrak{n}$, and $1 \leq p \leq D:=\frac{1}{2} q(q-1)$.

Choose a subspace $\mathfrak{b}$ complementary to $\mathfrak{m}$ in $\mathfrak{n}$ and two bases: $\left\{X_{i}\right\}$ for $\mathfrak{b}$ and $\left\{Z_{k}\right\}$ for $\mathfrak{m}$. The Lie bracket on $\mathfrak{n}$ defines (and is defined by) a $p$-tuple of skewsymmetric $q \times q$ matrices $J_{1}, \ldots, J_{p}$ such that $\left[X_{i}, X_{j}\right]=\sum_{\alpha=1}^{p}\left(J_{\alpha}\right)_{i j} Z_{\alpha}$. The space of such $p$-tuples is $\mathcal{V}(p, q)=\left(\bigwedge^{2} \mathbb{R}^{q}\right)^{p}$. Note that the $J_{\alpha}$ 's must be linearly independent, as $\mathfrak{m}=[\mathfrak{n}, \mathfrak{n}]$, so the points of $\mathcal{V}(p, q)$ corresponding to the algebras of type $(p, q)$ form a subset $\mathcal{V}^{0}(p, q) \subset \mathcal{V}(p, q)$, which is the complement to a real algebraic subset. The spaces $\mathcal{V}(p, q)$ and $\mathcal{V}^{0}(p, q)$ are acted upon by the group $\operatorname{GL}(q) \times \operatorname{GL}(p)$ (change of bases): for $x=\left(J_{1}, \ldots, J_{p}\right) \in \mathcal{V}(p, q)$ and $(M, T) \in$ $\operatorname{GL}(q) \times \operatorname{GL}(p),(M, T) \cdot x=\left(\tilde{J}_{1}, \ldots, \tilde{J}_{p}\right)$, with $\tilde{J}_{\alpha}=\sum_{\beta=1}^{p}\left(T^{-1}\right)_{\beta \alpha} M J_{\beta} M^{t}$ (note that instead of fixing the basis and deforming the Lie bracket, as in the action $g . \mu(X, Y)=g \mu\left(g^{-1} X, g^{-1} Y\right)$, we now keep the Lie bracket fixed and change the basis). Clearly, two points of $\mathcal{V}^{0}(p, q)$ lying on the same $\operatorname{GL}(q) \times \operatorname{GL}(p)$-orbit define isomorphic algebras. The converse is also true, so the space $\mathcal{X}(p, q)$ of the isomorphism classes of two-step nilpotent Lie algebras of type $(p, q)$ is the quotient space $\mathcal{V}^{0}(p, q) /(\operatorname{GL}(q) \times \operatorname{GL}(p))$. The space $\mathcal{X}(p, q)$ is compact but in general is non-Hausdorff.

By Proposition A of [Eb1, Section 5.4d], when $q \geq 6$ and $2<p<D-2$, or when $(p, q)=(5,5)$, the space $\mathcal{V}(p, q)$ contains no open $\operatorname{GL}(q) \times \mathrm{GL}(p)$-orbits; that is, no two-step nilpotent algebras of type $(p, q)$ are locally rigid (an open orbit of the action of $\operatorname{SL}(q)$ on the Grassmannian $G\left(p, \bigwedge^{2} \mathbb{R}^{q}\right)$ considered in Eb1] occurs exactly when the action of $\operatorname{GL}(q) \times \operatorname{GL}(p)$ on $\mathcal{V}(p, q)$ has an open orbit). 
It will be more convenient to consider the action of $\mathrm{SL}(q) \times \mathrm{SL}(p)$ rather than $\mathrm{GL}(q) \times \mathrm{GL}(p)$. The $\mathrm{SL}(q) \times \mathrm{SL}(p)$-orbits distinguish the isomorphism classes up to scaling, which is easy to control.

The splitting $\mathfrak{n}=\mathfrak{b} \oplus \mathfrak{m}$ of a two-step nilpotent Lie algebra $\mathfrak{n}$ is a gradation, which corresponds to the canonical derivation $\Psi$ defined by $\Psi(X+Z)=X+2 Z$, for any $X \in \mathfrak{b}, Z \in \mathfrak{m}$. If a pre-Einstein derivation $\phi$ for $\mathfrak{n}$ is proportional to $\Psi$, then the group $G_{\phi}$ from Theorem 2 is precisely $\operatorname{SL}(q) \times \operatorname{SL}(p)$ (see (9)). If, in addition, $\mathfrak{n}$ is an Einstein nilradical, then it has the eigenvalue type $(1,2 ; q, p)$. Thus Theorem 2 implies the following:

Proposition 1 ([La3, Proposition 9.1]). A two-step nilpotent Lie algebra $\mathfrak{n}$ of type $(p, q)$ corresponding to a point $x \in \mathcal{V}(p, q)$ is an Einstein nilradical of the eigenvalue type $(1,2 ; q, p)$ if and only if the orbit $(\mathrm{SL}(q) \times \mathrm{SL}(p)) . x \subset \mathcal{V}(p, q)$ is closed.

Note that if a pre-Einstein derivation of a nilpotent Lie algebra $\mathfrak{n}$ is proportional to the one having only eigenvalues 1 and 2 , then $\mathfrak{n}$ is automatically two-step nilpotent. Theorem 4 says that a typical algebra with such a pre-Einstein derivation is an Einstein nilradical.

Let $\mathfrak{n}$ be a two-step nilpotent Lie algebra of type $(p, q)$, with $1 \leq p<D$, defined by a point $x=\left(J_{1}, \ldots, J_{p}\right) \in \mathcal{V}^{0}(p, q)$. Choose an arbitrary basis $J_{\alpha}^{\prime}, \alpha=1, \ldots, D-$ $p$, in the orthogonal complement to the subspace $\operatorname{Span}\left(J_{1}, \ldots, J_{p}\right) \subset \bigwedge^{2} \mathbb{R}^{q}$ with respect to the inner product $Q\left(K_{1}, K_{2}\right)=-\operatorname{Tr}\left(K_{1} K_{2}\right)$ on $\bigwedge^{2} \mathbb{R}^{q}$. The point $x^{\prime}=$ $\left(J_{1}^{\prime}, \ldots, J_{D-p}^{\prime}\right) \in \mathcal{V}^{0}(D-p, q)$ defines a two-step nilpotent Lie algebra $\mathfrak{n}^{*}$ of type $(D-p, q)$, which is called the dual to $\mathfrak{n}$. It is easy to check that the isomorphism class of $\mathfrak{n}^{*}$ is well-defined (depends only on the isomorphism class of $\mathfrak{n}$ ).

Proof of Theorem 4. Let a pair $(p, q)$ with $1 \leq p \leq q(q-1) / 2$ be such that the stabilizer in general position (the s.g.p.) of the group $G^{\mathbb{C}}=\operatorname{SL}(q, \mathbb{C}) \times \operatorname{SL}(p, \mathbb{C})$ acting on the space $\mathcal{V}^{\mathbb{C}}(p, q)=\left(\bigwedge^{2} \mathbb{C}^{q}\right)^{p}$ is reductive. This means that there exists a reductive complex Lie algebra $\mathfrak{h}$ and a nonempty $G^{\mathbb{C}}$-invariant Zariski-open subset $U^{\prime} \subset \mathcal{V}^{\mathbb{C}}(p, q)$ such that for any point $x \in U^{\prime}$ the Lie algebra of the stabilizer $G_{x}^{\mathbb{C}}$ is isomorphic to $\mathfrak{h}[\mathrm{VP}, \S 7]$.

By [Ela, in most cases the s.g.p. of the action of $G^{\mathbb{C}}$ on $\mathcal{V}^{\mathbb{C}}(p, q)$ is finite; that is, $\mathfrak{h}=0$ (as it is suggested by the dimension count). The cases when it is not are listed in Table 6 of Ela. Examining that table we see that the s.g.p. is reductive unless $(p, q)=(2,2 k+1)$.

By the Popov criterion $\mathrm{Pop}$, in all the cases when the s.g.p. is reductive, the action is stable; that is, there exists a nonempty Zariski-open subset $U^{\prime \prime} \subset \mathcal{V}^{\mathbb{C}}(p, q)$, which is a union of closed $G^{\mathbb{C}}$-orbits. Let $U$ be the set of real points of $U^{\prime \prime} \cap U^{\prime}$. Then $U$ is a $\operatorname{SL}(q) \times \operatorname{SL}(p)$-invariant semialgebraic subset of $\mathcal{V}(p, q)=\left(\bigwedge^{2} \mathbb{R}^{q}\right)^{p}$ and is open and dense in the Euclidean topology in $\mathcal{V}(p, q)$.

It now follows from Remark 4 and Proposition 1 that for every point $x \in U, x \neq$ 0 , the two-step nilpotent Lie algebra corresponding to $x$ is an Einstein nilradical. Note that every such $x$ lies in $\mathcal{V}^{0}(p, q)$, hence defining a two-step nilpotent Lie algebra $\mathfrak{n}$ precisely of type $(p, q)$ (for if $x=\left(J_{1}, \ldots, J_{p}\right) \in U$ and the $J_{\alpha}$ 's are linearly dependent, then the closure of the orbit $(\mathrm{SL}(q) \times \mathrm{SL}(p)) \cdot x$ contains the origin of $\mathcal{V}(p, q))$. Hence the eigenvalue type of the Einstein nilradical $\mathfrak{n}$ is $(1,2 ; q, p)$.

The proof shows that a generic point of $\mathcal{V}(p, q)$ defines an Einstein nilradical with the eigenvalue type $(1,2 ; q, p)$ in all the cases except for $(p, q)=(2,2 k+1)$ (in fact, 
there are no two-step Einstein nilradicals of type $(1,2 ; 2 k+1,2)$ at all, as follows from [GK, Proposition 2.9(v)]). In Theorem 4 we narrow the dimension range to exclude those cases when some algebras of type $(p, q)$ have open orbits in $\mathcal{V}(p, q)$ (and additionally, the cases $(p, q)=(2,2 k), k>3)$. The remaining cases give a reasonable notion of being typical, not only in the linear space $\mathcal{V}(p, q)$, but also in the non-Hausdorff space $\mathcal{X}(p, q)$ of isomorphism classes of two-step nilpotent algebras of type $(p, q)$. Note that, in general, the condition of typicality of a nilpotent Lie algebra could hardly be nicely defined (see [uk]). On the other hand, taking the categorical quotient $\mathcal{V}(p, q) / /(\mathrm{SL}(q) \times \mathrm{SL}(p))$ is somewhat tautological in view of the proof of Theorem 4 .

Theorem 4 omits two-step nilpotent Lie algebras $\mathfrak{n}$ with the following $(p, q)$ :

- $p=1$. Any such $\mathfrak{n}$ is the direct sum of a Heisenberg algebra and an abelian ideal and is an Einstein nilradical (the corresponding solvmanifold can be taken as the product of a real and a complex hyperbolic space).

- $p=D$, a free two-step nilpotent algebra; $\mathfrak{n}$ is an Einstein nilradical by GK, Proposition 2.9].

- $p=D-1$. Any such algebra is an Einstein nilradical by [Ni3, Proposition 3].

- $p=2, p=D-2$. These algebras can be completely classified using the Kronecker theory of matrix pencils. The approach suggested at the end of Section 3 can be used to find Einstein nilradicals among them.

- $q \leq 5,(p, q) \neq(5,5)$. Using Theorem 3 and the classification from [GT], we find below all the Einstein nilradicals among these algebras.

First of all, by Wil, Theorem 3.1] and La2, Theorem 5.1], any nilpotent Lie algebra of dimension six or lower is an Einstein nilradical (no longer true in dimension 7). Second, any two-step nilpotent algebra with $p=1, D$, or $D-1$ is an Einstein nilradical. This leaves out the following list of pairs $(p, q)$ :

$$
(p, q)=(3,4),(4,4),(2,5),(3,5),(4,5),(6,5),(7,5),(8,5) .
$$

According to the classification in GT, Table 2], all the algebras with $(p, q)=$ $(3,4),(4,4),(2,5),(3,5)$, and $(4,5)$ over $\mathbb{C}$ fall into a finite number of classes, each of which is defined over $\mathbb{R}$. The same is true for the remaining three cases $(p, q)=(6,5),(7,5),(8,5)$, which give the algebras dual to the algebras of types $(p, q)=(4,5),(3,5)$, and $(2,5)$, respectively. Note that the dual to an Einstein nilradical is not necessarily an Einstein nilradical.

By Theorem [6, it suffices to consider the algebras in [GT, Table 2] and their duals as the real Lie algebras. The majority of them (all five of type $(3,4)$, all three of type $(4,4)$, all five of type $(2,5), 15$ out of 17 of type $(3,5)$, and 31 out of 38 of type $(4,5))$ have a nice basis, which is the basis given in the table. Moreover, as $q \leq 5$, the two-step nilpotent Lie algebra dual to one having a nice basis also has a nice basis, which easily follows from Definition 3 .

Therefore, for all but two algebras of type $(3,5)$, seven algebras of type $(4,5)$ and their duals (two of type $(7,5)$ and seven of type $(6,5)$ ), the question of whether the algebra is an Einstein nilradical is completely answered by Theorem 3 , It turns out that in all these cases the matrix $Y$ has the maximal rank $m$, so that the equation $Y Y^{t} \alpha=[1]_{m}$ from (ii) of Theorem 3 has a unique solution $\alpha$. A direct computation shows that the only cases when the algebra fails to be an Einstein nilradical are the following: three algebras of type $(7,5)$ dual to the algebras $\mathbf{7 5}, \mathbf{8 7}$ and $\mathbf{1 0 2}$, and six algebras of type $(6,5)$ dual to the algebras $21,36,41,50,52$ and 59 from 
TABLE 1. Two-step nilpotent Lie algebras of types $(3,5)$ and $(4,5)$ for which the basis $\left\{X_{i}, Z_{\alpha}\right\}$ is not a nice basis

\begin{tabular}{|c|l|l|}
\hline $\mathbf{2 6}$ & \multicolumn{1}{|c|}{ Relations } & \multicolumn{1}{|c|}{ Orthonormal basis } \\
\hline $\mathbf{2 8}$ & $133,152,233,244,251,341$ & $\frac{-1}{\sqrt{3}}\left(2 X_{1}-X_{2}\right), \frac{\sqrt{2}}{\sqrt{3}} X_{2}, X_{3}, X_{4}, X_{5}$, \\
& $134,143,152,233,242,251,342$ & $Z_{3}, Z_{4}, \frac{-\sqrt{3}}{\sqrt{10}}\left(Z_{1}+2 Z_{2}\right), \frac{\sqrt{3}}{\sqrt{2}} Z_{2}$ \\
& & $2 \sqrt{3} X_{1},-2 X_{2}+X_{3}, \sqrt{6} X_{3}, \sqrt{6} X_{4}, X_{5}$, \\
& $Z_{4}, Z_{3}, \frac{1}{\sqrt{2}} Z_{2}, \frac{1}{\sqrt{30}} Z_{1}$ \\
$\mathbf{4 4}$ & $124,143,152,232,242,351$ & $\sqrt{6} X_{1}, \sqrt{6} X_{2},-2 X_{3}+X_{4}, \sqrt{6} X_{4}, X_{5}$, \\
& & $Z_{4}, Z_{3}, Z_{2}, \frac{1}{\sqrt{15}} Z_{1}$ \\
$\mathbf{4 5}$ & $123,142,151,232,243,344$ & $\frac{1}{\sqrt{6}}\left(2 X_{1}+X_{4}\right), 2 X_{2}, X_{3}, X_{4}, \frac{\sqrt{5}}{\sqrt{2}} X_{5}$, \\
& & $Z_{4}, 2 Z_{3}, 2 Z_{2}, Z_{1}$ \\
$\mathbf{5 5}$ & $124,132,142,243,351$ & $X_{1}, X_{2}, \frac{1}{\sqrt{6}}\left(-2 X_{3}+X_{4}\right), X_{4}, X_{5}$, \\
& & $Z_{4}, Z_{3}, Z_{2}, \frac{\sqrt{2}}{\sqrt{5}} Z_{1}$ \\
$\mathbf{6 0}$ & $124,132,143,232,251,341$ & $\frac{1}{\sqrt{2}}\left(-2 X_{1}+X_{2}\right), \frac{\sqrt{3}}{\sqrt{2}} X_{2}, X_{3}, X_{4}, X_{5}$, \\
& & $\sqrt{3} Z_{4}, Z_{3}, Z_{2}, Z_{1}$ \\
$\mathbf{6 6}$ & $124,131,153,231,242$ & $-2 X_{1}+X_{2}, \sqrt{3} X_{2}, X_{3}, X_{4}, X_{5}$, \\
& & $\sqrt{12} Z_{4}, Z_{3}, Z_{2}, Z_{1}$ \\
$\mathbf{7 2}$ & $132,143,232,251,341$ & $X_{1}+X_{2}, \sqrt{3}\left(X_{1}-X_{2}\right), X_{3}, X_{4}, X_{5}$, \\
& & $Z_{3}, Z_{2}, Z_{1}$ \\
$\mathbf{7 8}$ & $131,153,231,242$ & $X_{1}+X_{2}, \sqrt{3}\left(X_{1}-X_{2}\right), X_{3}, X_{4}, X_{5}$, \\
& & $Z_{1}, Z_{2}, Z_{3}$ \\
\hline
\end{tabular}

GT, Table 2]. Perhaps the most interesting of them is algebra 102: viewed as an algebra of type $(3,5)$, it is the direct sum of the free two-step nilpotent algebra on three generators and a two-dimensional abelian ideal.

In the remaining cases, when the basis given in GT is not nice, we follow steps (a) and (c) at the end of Section 3, first finding a pre-Einstein derivation and then solving the system of equations for the nilsoliton metric. All nine algebras $\mathbf{2 6}, \mathbf{2 8}, \mathbf{4 4}, \mathbf{4 5}, \mathbf{5 5}, \mathbf{6 0}, \mathbf{6 6}, \mathbf{7 2}, \mathbf{7 8}$ and their duals appear to be Einstein nilradicals. An explicit form of the nilsoliton inner product is given in Tables 1 and 2, where we use the following notation. The first column is the number of the algebra in the list GT, Table 2], and the asterisk means the dual algebra. The column "Relations" lists the nonzero brackets with respect to the basis $\left\{X_{i}, Z_{\alpha}\right\}$. For instance, algebra 28* is defined by the relations $\left[X_{1}, X_{2}\right]=Z_{1},\left[X_{1}, X_{5}\right]=-Z_{3}-Z_{4},\left[X_{2}, X_{3}\right]=Z_{2}$, $\left[X_{1}, X_{4}\right]=-Z_{2},\left[X_{2}, X_{4}\right]=Z_{3},\left[X_{3}, X_{4}\right]=Z_{4},\left[X_{3}, X_{5}\right]=Z_{5}$, and $\left[X_{4}, X_{5}\right]=Z_{6}$. The third column gives an orthonormal basis for a nilsoliton inner product (which can be checked directly by (5) and (6) ). Note that the algebras from Tables 1 and 2 exhibit quite exotic eigenvalue types.

Summarizing these results, we obtain the following proposition.

Proposition 2. A two-step nilpotent Lie algebra of type $(p, q)$, with $q \leq 5$ and $(p, q) \neq(5,5)$, is an Einstein nilradical, unless it is isomorphic (over $\mathbb{C})$ to an 11dimensional algebra of type $(6,5)$ dual to one of the algebras $\mathbf{2 1}, \mathbf{3 6}, \mathbf{4 1}, \mathbf{5 0}, \mathbf{5 2}, \mathbf{5 9}$, 
or to a 12-dimensional algebra of type $(7,5)$ dual to one of the algebras $\mathbf{7 5 , 8 7}$, or 102 from [GT, Table 2].

TABLE 2. The algebras dual to the algebras from Table 1

\begin{tabular}{|c|c|c|}
\hline & Relations & Orthonormal basis \\
\hline $26^{*}$ & $\begin{array}{l}-251,341,232 \\
-132,123,144 \\
355,456\end{array}$ & $\begin{array}{l}-\frac{\sqrt{3}}{\sqrt{91}}\left(26 X_{1}+19 X_{2}\right),-\frac{3 \sqrt{209}}{\sqrt{91}} X_{2}, \frac{12 \sqrt{19}}{7} X_{3}, \frac{11}{6} X_{4}, \frac{\sqrt{22}}{\sqrt{3}} X_{5}, \\
Z_{1}, \frac{6 \sqrt{6}}{\sqrt{77}} Z_{2}, Z_{3}, \frac{\sqrt{627}}{16 \sqrt{14}} Z_{4}, Z_{5}, \frac{77}{72 \sqrt{19}} Z_{6}\end{array}$ \\
\hline $28^{*}$ & $\begin{array}{l}121,-153-154 \\
232,-142,243 \\
344,355,456\end{array}$ & $\begin{array}{l}\frac{8 \sqrt{266}}{\sqrt{5}} X_{1}, \frac{12 \sqrt{57}}{\sqrt{145}} X_{2}, \frac{1}{\sqrt{29}}\left(9 X_{2}+29 X_{3}\right), X_{4}, \frac{\sqrt{3}}{2 \sqrt{14}} X_{5}, \frac{16 \sqrt{14}}{5} Z_{1} \\
\frac{4 \sqrt{2}}{\sqrt{5}} Z_{2}, \frac{4 \sqrt{6}}{\sqrt{145}} Z_{3}, \frac{1}{19 \sqrt{29}}\left(-9 Z_{3}+29 Z_{4}\right), \frac{\sqrt{3}}{10 \sqrt{7}} Z_{5}, \frac{\sqrt{3}}{16 \sqrt{266}} Z_{6}\end{array}$ \\
\hline $44^{*}$ & $\begin{array}{l}131,-152-153 \\
232,243,254 \\
345,456\end{array}$ & $\begin{array}{l}\frac{2 \sqrt{199}}{3 \sqrt{145}} X_{1}, \frac{1}{\sqrt{29}} X_{2}, \frac{\sqrt{2}}{3 \sqrt{2805}}\left(X_{3}+\frac{33}{58} X_{4}\right), \frac{1}{29} X_{4}, \frac{1}{\sqrt{5771}} X_{5}, Z_{6} \\
\frac{398 \sqrt{2}}{3 \sqrt{33}} Z_{1}, \frac{10 \sqrt{34}}{\sqrt{33}} Z_{2}, \frac{-\sqrt{65}}{78}\left(154 Z_{2}+199 Z_{3}\right), \frac{5 \sqrt{17}}{\sqrt{23}} Z_{4}, \frac{2 \sqrt{995}}{\sqrt{1353}} Z_{5}\end{array}$ \\
\hline $45^{*}$ & $\begin{array}{l}-141,231,242 \\
-122,133,254 \\
355,456\end{array}$ & $\begin{array}{l}13 X_{1}-8 X_{4}, \frac{2}{5} X_{2}, 260 X_{3}, 12 X_{4}, X_{5}, \sqrt{130} Z_{1}, \frac{1}{\sqrt{10}} Z_{2} \\
65 \sqrt{10} Z_{3}, \frac{1}{10 \sqrt{13}} Z_{4}, 5 \sqrt{13} Z_{5}, Z_{6}\end{array}$ \\
\hline $55^{*}$ & $\begin{array}{l}-131,141,152 \\
233,254,345 \\
456\end{array}$ & $\begin{array}{l}X_{1}, X_{2}, \frac{1}{2 \sqrt{6}}\left(17 X_{3}+12 X_{4}\right), \sqrt{11} X_{4}, X_{5}, \frac{\sqrt{34}}{2 \sqrt{3}} Z_{1}, \frac{\sqrt{5}}{\sqrt{7}} Z_{2} \\
\frac{\sqrt{34}}{2 \sqrt{3}} Z_{3}, \frac{\sqrt{5}}{\sqrt{7}} Z_{4}, \frac{17 \sqrt{55}}{2 \sqrt{39}} Z_{5}, \sqrt{17} Z_{6}\end{array}$ \\
\hline $60^{*}$ & $\begin{array}{l}-251,341,232 \\
-132,153,244 \\
355,456\end{array}$ & $\begin{array}{l}\sqrt{19}\left(2 X_{1}+X_{2}\right), 3 \sqrt{3} X_{2}, 4 X_{3}, \sqrt{33} X_{4}, \sqrt{22} X_{5}, Z_{1}, \frac{4}{\sqrt{33}} Z_{2} \\
\frac{\sqrt{19}}{\sqrt{51}}\left(-Z_{1}+2 Z_{3}\right), Z_{4}, \frac{4}{\sqrt{33}} Z_{5}, Z_{6}\end{array}$ \\
\hline $66^{*}$ & $\begin{array}{l}-131,231,452 \\
143,254,345 \\
356\end{array}$ & $\frac{1}{\sqrt{2}}\left(2 X_{1}+X_{2}\right), \frac{\sqrt{3}}{\sqrt{2}} X_{2}, X_{3}, X_{4}, X_{5}, Z_{1}, Z_{2}, Z_{3}, Z_{4}, Z_{5}, Z_{6}$ \\
\hline $72^{*}$ & $\begin{array}{l}-131,231,342 \\
-252,123,154 \\
245,356,457\end{array}$ & $\begin{array}{l}4 X_{1}+2 X_{2}, \frac{\sqrt{19}}{\sqrt{2}} X_{2}, \frac{1}{3} X_{3}, \sqrt{11} X_{4}, \frac{1}{\sqrt{57}} X_{5}, Z_{1}, Z_{2}, \frac{12 \sqrt{19}}{\sqrt{29}} Z_{3} \\
\frac{2 \sqrt{6}}{5 \sqrt{19}}\left(Z_{2}-2 Z_{4}\right), 3 \sqrt{11} Z_{5}, \frac{\sqrt{2}}{\sqrt{1311}} Z_{6}, \frac{\sqrt{66}}{\sqrt{437}} Z_{7}\end{array}$ \\
\hline $78^{*}$ & $\begin{array}{l}-131,231,122 \\
143,254,345 \\
356,457\end{array}$ & $\begin{array}{l}-2 \sqrt{3}\left(X_{1}+X_{2}\right), 2\left(X_{1}-X_{2}\right), \sqrt{14} X_{3}, \sqrt{51} X_{4}, \sqrt{51} X_{5} \\
\frac{\sqrt{14}}{\sqrt{51}} Z_{1}, \frac{4 \sqrt{2}}{3 \sqrt{17}} Z_{2}, Z_{3}, Z_{4}, Z_{5}, Z_{6}, \frac{\sqrt{51}}{\sqrt{14}} Z_{7}\end{array}$ \\
\hline
\end{tabular}

\section{REFERENCES}

[Al1] Alekseevskii D.V., Classification of quaternionic spaces with transitive solvable group of motions, Izv. Akad. Nauk SSSR Ser. Mat., 39 (1975), 315 - 362. MR0402649 (53:6465)

[Al2] _ Homogeneous Riemannian spaces of negative curvature, Mat. Sb. (N.S.), 96(138) (1975), 93 - 117. MR0362145 (50:14587)

[AK] Alekseevskii D.V., Kimel'fel'd B.N., Structure of homogeneous Riemannian spaces with zero Ricci curvature, Funct. Anal. Appl., 9 (1975), 97 - 102. MR 0402650 (53:6466)

[Bir] Birkes D., Orbits of linear algebraic groups, Ann. of Math. (2), 93 (1971), 459 - 475. MR 0296077 (45:5138) 
[BHC] Borel A., Harish-Chandra, Arithmetic Subgroups of Algebraic Groups, Ann. of Math. (2), 75 (1962), 485 - 535. MR0147566 (26:5081)

[DM] Dotti Miatello I., Ricci curvature of left-invariant metrics on solvable unimodular Lie groups, Math. Z., 180 (1982), 257 - 263. MR0661702 (84a:53044)

[Eb1] Eberlein P., The moduli space of 2-step nilpotent Lie algebras of type $(p, q)$, Explorations in complex and Riemannian geometry, Contemp. Math., 332, Amer. Math. Soc., Providence, RI, 2003, 37 - 72. MR2016090 (2004j:17015)

[Eb2] _ Geometry of 2-step nilpotent Lie groups, Modern Dynamical Systems and applications, Cambridge Univ. Press, Cambridge, 2004, 67 - 101. MR2090766 (2005m:53081)

[Eb3] _ Riemannian 2-step nilmanifolds with prescribed Ricci tensor, Geometric and Probabilistic Structures in Dynamics, Contemp. Math., 469, Amer. Math. Soc., Providence, RI, 2008, 167 - 195. MR2478470 (2010c:53074)

[Ela] Elashvili A.G., Stationary subalgebras of points of general position for irreducible linear Lie groups, Funct. Anal. Appl., 6 (1972), 139 - 148. MR0304555 (46:3690)

[GK] Gordon C., Kerr M., New homogeneous Einstein metrics of negative Ricci curvature, Ann. Global Anal. Geom., 19 (2001), 75 - 101. MR.1824172 (2002f:53067)

[GT] Galitski L., Timashev D., On classification of metabelian Lie algebras, J. Lie Theory, 9 (1999), 125 - 156. MR:1680007 (2000f:17015)

[Heb] Heber J., Noncompact homogeneous Einstein spaces, Invent. Math., 133 (1998), 279 352. MR1632782 (99d:53046)

[La1] Lauret J., Ricci soliton homogeneous nilmanifolds, Math. Ann., 319 (2001), 715 - 733. MR 1825405 (2002k:53083)

[La2] - Finding Einstein solvmanifolds by a variational method, Math. Z., 241 (2002), 83 - 99. MR.1930986 (2003g:53064)

[La3] Degenerations of Lie algebras and geometry of Lie groups, Differential Geom. Appl., 18 (2003), 177 - 194. MR1958155 (2004c:17008)

[La4] On the moment map for the variety of Lie algebras, J. Funct. Anal., 202 (2003), 392 - 423. MR.1990531 (2004d:14067)

[La5] _ Einstein solvmanifolds are standard, Ann. of Math. (2) 172 (2010), 1859 - 1877. MR 2726101

[LW] Lauret J., Will C., Einstein solvmanifolds: Existence and non-existence questions, preprint 2006, arXiv: math.DG/0602502.

[Luk] Luks E.M., What is the typical nilpotent Lie algebra? Computers in nonassociative rings and algebras, 189 - 207. Academic Press, New York, 1977. MR0453830 (56:12083)

[Mil] Milnor J., Curvatures of left invariant metrics on Lie groups, Adv. Math. 21 (1976), 293 - 329. MR0425012 (54:12970)

[Mos] Mostow G.D., Fully reducible subgroups of algebraic groups, Amer. J. Math., 78 (1956), 200 - 221. MR0092928(19:1181f)

[Ni1]_ Einstein solvmanifolds with a simple Einstein derivation, Geom. Dedicata, 135 (2008), 87 - 102. MR2413331

[Ni2] Einstein solmanifolds with free nilradical, Ann. Global Anal. Geom., 33 (2008), 71 - 87. MR2369187 (2008m:53120)

[Ni3] _ Einstein solvmanifolds attached to two-step nilradicals, preprint, 2008, arXiv: math.DG/0805.0646.

[Nik] Nikonorov Yu.G., On Einstein extensions of nilpotent metric Lie algebras, Siberian Adv. Math., 17 (2007), 153 - 170. MR2647826

[Pay] Payne T., The existence of soliton metrics for nilpotent Lie groups, Geom. Dedicata, 145 (2010), 71-88. MR2600946

[Pop] Popov V.L., Criteria for the stability of the action of a semisimple group on the factorial of a manifold, Izv. Akad. Nauk SSSR Ser. Mat., 34 (1970), 523 - 531. MR0262416 (41:7024)

[RS] Richardson R., Slodowy P., Minimum vectors for real reductive algebraic groups, J. London Math. Soc. (2), 42 (1990), 409 - 429. MR1087217 (92a:14055)

[RWZ] Rand D., Winternitz P., Zassenhaus H., On the identification of a Lie algebra given by its structure constants. I. Direct decompositions, Levi decompositions, and nilradicals, Linear Algebra Appl., 109 (1988), 197 - 246. MR0961578 (89i:17001)

[VP] Vinberg E.B., Popov V.L., Invariant theory. In: Algebraic geometry. IV. Encyclopaedia of Mathematical Sciences, 55, Springer-Verlag, Berlin, 1994. MR1100485 (92d:14010) 
[Wil] Will C., Rank-one Einstein solvmanifolds of dimension 7, Differential Geom. Appl., 19 (2003), 307 - 318. MR2013098 (2004j:53060)

[WZ] Wolf J.A., Zierau R., Riemannian exponential maps and decompositions of reductive Lie groups, Topics in geometry, Progr. Nonlinear Differential Equations Appl., 20, Birkhäuser Boston, Boston, 1996, 349-353. MR 1390323 (97c:53080)

Department of Mathematics, La Trobe University, Victoria, 3086, Australia

E-mail address: y.nikolayevsky@latrobe.edu.au 